ARTICLE

\title{
FNDC4 acts as an anti-inflammatory factor on macrophages and improves colitis in mice
}

\author{
Madeleen Bosma1, Marco Gerling², Jenny Pasto ${ }^{1}$, Anastasia Georgiadi', Evan Graham¹, Olga Shilkova1, \\ Yasunori Iwata ${ }^{3}$, Sven Almer ${ }^{4,5}$, Jan Söderman ${ }^{6}$, Rune Toftgård ${ }^{2}$, Fredrik Wermeling ${ }^{4}$, Elisabeth Almer Boström \\ \& Pontus Almer Boström
}

FNDC4 is a secreted factor sharing high homology with the exercise-associated myokine irisin (FNDC5). Here we report that Fndc4 is robustly upregulated in several mouse models of inflammation as well as in human inflammatory conditions. Specifically, FNDC4 levels are increased locally at inflamed sites of the intestine of inflammatory bowel disease patients. Interestingly, administration of recombinant FNDC4 in the mouse model of induced colitis markedly reduces disease severity compared with mice injected with a control protein. Conversely, mice lacking Fndc4 develop more severe colitis. Analysis of binding of FNDC4 to different immune cell types reveals strong and specific binding to macrophages and monocytes. FNDC4 treatment of bone marrow-derived macrophages in vitro results in reduced phagocytosis, increased cell survival and reduced proinflammatory chemokine expression. Hence, treatment with FNDC4 results in a state of dampened macrophage activity, while enhancing their survival. Thus, we have characterized FNDC4 as a factor with direct therapeutic potential in inflammatory bowel disease and possibly other inflammatory diseases.

\footnotetext{
${ }^{1}$ Department of Cell and Molecular Biology, Karolinska Institutet, Stockholm SE-171 77, Sweden. ${ }^{2}$ Department of Biosciences and Nutrition, Center of Innovative Medicine, Karolinska Institutet, Huddinge SE-141 83, Sweden. ${ }^{3}$ Division of Nephrology, Kanazawa University Hospital, Kanazawa $920-8641$, Japan. ${ }^{4}$ Department of Medicine, Solna, Karolinska Institutet, and Karolinska University Hospital, Stockholm SE-171 76, Sweden. ${ }^{5}$ GastroCentrum, Karolinska University Hospital, Solna, Stockholm SE-171 76, Sweden. ${ }^{6}$ Division of Medical Diagnostics, Ryhov County Hospital, Jönköping 55185, Sweden. ${ }^{7}$ Department of Dental Medicine, Karolinska Institutet, Huddinge SE-141 83, Sweden. Correspondence and requests for materials should be addressed to M.B. (email: madeleen.bosma@ki.se).
} 
$\mathrm{T}$ he incidence and prevalence of Crohn's disease and ulcerative colitis, the two major forms of inflammatory bowel disease (IBD), are rising globally ${ }^{1}$. IBD is characterized by inflammation of the gastrointestinal tract of genetically susceptible individuals exposed to environmental factors ${ }^{2}$. The hallmark of the pathogenesis is a hyperactive and deregulated immune function in the bowel, where macrophages play a central role ${ }^{3}$. Treatment possibilities are limited and often come at the cost of potentially severe side effects ${ }^{4}$. Therefore, further knowledge on the molecular pathology and the development of novel treatment approaches is highly warranted.

We have characterized a novel secreted factor, FNDC4, a member of the fibronectin type III domain family of proteins. This protein family consists of five proteins (FNDC1-5), of which FNDC4 shows the strongest homology with FNDC5 (ref. 5). FNDC5/irisin was recently identified as an exercise-induced secreted factor targeting adipose tissue to induce brown fat formation and energy expenditure ${ }^{6}$. Irisin is released from skeletal muscle in response to exercise by cleavage of its precursor, the type 1 membrane protein FNDC5 (refs 6,7). Conclusive data now show that irisin protein circulates in humans, at concentrations typical for hormones ${ }^{8}$. Irisin is a soluble polypeptide consisting primarily of one fibronectin type III domain ${ }^{6-8}$. Among the members of proteins containing the fibronectin type III domain, this domain architecture is only found in one additional gene: Fndc4 (also known as Frcp1) (ref. 5). FNDC4 protein shares 57\% amino acid identity with FNDC5 in the functional, extracellular domain. Like Fndc5, Fndc4 is highly conserved (excluding the signal peptide, human FNDC4 is $100 \%$ homologous to mouse Fndc4). Fndc4 expression is highest in liver and brain, but it is expressed at lower levels in many other tissues, including heart, skeletal muscle and adipose tissue.

The observation that Fndc4, but not Fndc5, is robustly regulated in the inflamed state led us to investigate its potential role in immunomodulation. Here we show that administration of FNDC4 results in reduced inflammation and disease severity in a mouse model for IBD, indicating that FNDC4 could have therapeutic potential. FNDC4 binds to and induces signalling in one key inflammatory cell; the macrophage. Interestingly, FNDC4 fundamentally alters the activation state of macrophages, which has important consequences for macrophage function.

\section{Results}

FNDC4 is cleaved and secreted. Given its similarity to FNDC5, we investigated whether FNDC4 is cleaved to release a secreted part similar to FNDC5 processing. For this purpose, an N-terminal FLAG-tagged and C-terminal Myc-tagged FNDC4 construct was synthesized (Fig. 1a). After overexpression of the construct in HEK293 cells, a clear FLAG-positive protein band was identified in both cells and media when probing the N-terminal FLAG tag using western blot (Fig. 1b, left panel). Probing against Myc, however, resulted in a detectable band only in the cellular fraction (Fig. 1b, right panel). Thus, an N-terminal portion of FNDC4 is released from the cells in accordance with the release mechanism of FNDC5/irisin 6 .7. Importantly, the band in the media fraction was of reduced MW compared with the cellular version, signifying a cleaved version. Thus, the $\mathrm{N}$-terminus of FNDC4 can be shredded to release an extracellular portion of the protein.

Fndc4 is upregulated in settings of inflammatory disease. To explore the potential roles of FNDC4 and FNDC5 in inflammation, we first studied the expression of both Fndc4 and its close homologue Fndc5 in three models of inflammation; (i) dextran sodium sulfate (DSS)-induced inflammation of the colon, a

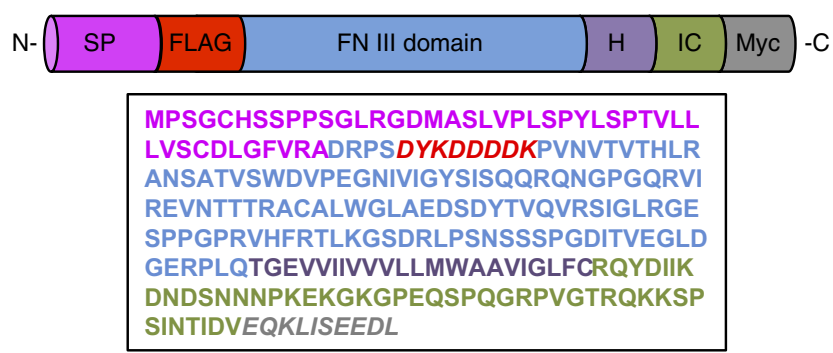

b

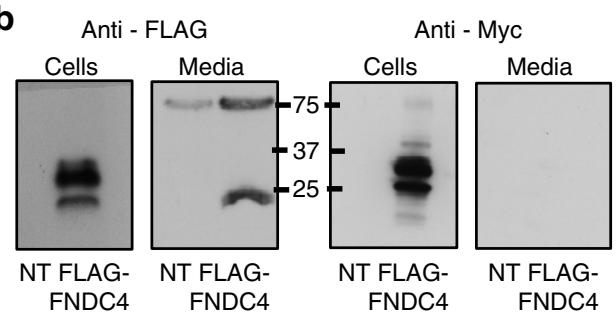

Figure 1 | An N-terminal portion of FNDC4 is secreted. (a) Graphical representation of the FNDC4 construct with N-terminal FLAG and C-terminal Myc tags as well as the peptide sequence. $\mathrm{C}$, intracellular domain; FN III domain, Fibronectin type III domain; $\mathrm{H}$, hydrophobic domain; $\mathrm{SP}$, signal peptide. (b) Western blot against either FLAG or Myc, as indicated, in cell- and media samples from HEK293 cells transfected with the N-terminal FLAG and C-terminal Myc FNDC4 construct. The full-size blot image is presented in Supplementary Fig. 11a. Similar results were seen in at least three independent experiments. NT, non-transfected control.

(ii) autoimmune inflammation of the kidney and (iii) ischaemiainduced inflammation of the kidney. Histology and gene expression data showing the degree of inflammation are presented in Supplementary Fig. 1. Interestingly, in all three models, Fndc4 gene expression was upregulated, whereas $F n d c 5$ was either downregulated or unchanged (Fig. 2a,b and Supplementary Fig. 2a,b). Moreover, with ischaemia reperfusion, the regulation of Fndc4 was seen both locally in the ischaemic kidney (Supplementary Fig. 2a), as well as in the liver (Supplementary Fig. 2c). In contrast to Fndc5, Fndc4 is not regulated by exercise training, at least not at the transcriptional level (Supplementary Fig. 2d,e). We concluded that Fndc4 and Fndc5 have clearly different expression profiles when comparing exercise and inflammation. Given the consistent upregulation of Fndc4 in inflammation and the fact that FNDC4 shows superior protein stability when produced as a recombinant protein compared with irisin/FNDC5, we focused subsequent studies on this molecule.

Subjects with IBD display higher FNDC4 expression. To investigate whether the inflammation-induced regulation of Fndc4 in mice translates to humans, we analysed FNDC4 and FNDC5 expression in patients with IBD and controls without intestinal inflammation. Intestinal biopsies obtained during ileocolonoscopy from 52 IBD patients (21 with Crohn's disease, 29 with ulcerative colitis and 2 with unclassified IBD, Supplementary Table 2) and 19 controls were analysed for FNDC4 and FNDC5 expression using quantitative reverse transcription-PCR (RT-qPCR). Paired samples from both non-inflamed and inflamed regions were available from 21 IBD patients ( 7 with Crohn's disease, 12 with ulcerative colitis and 2 with unclassified IBD). FNDC5 gene expression was undetectable in the intestinal biopsies. No gender differences or regional differences between ileum and colon or within different segments of the colon in FNDC4 expression were observed $(P=0.125$; 
a

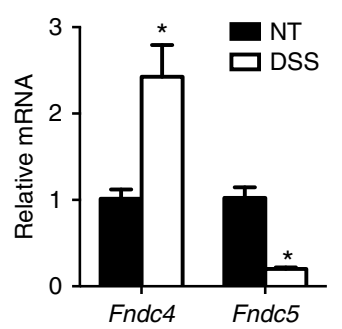

b

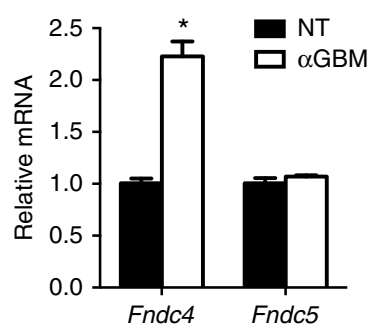

C

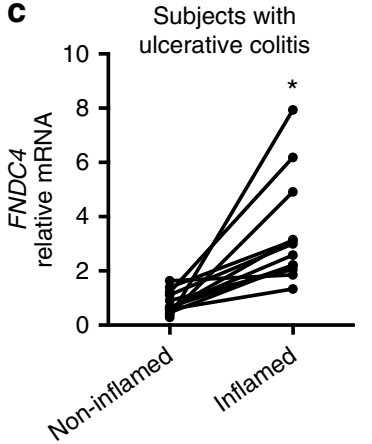

d

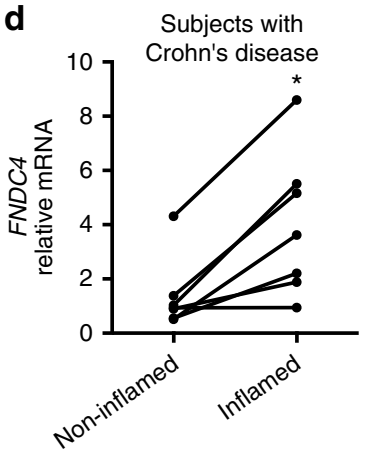

e

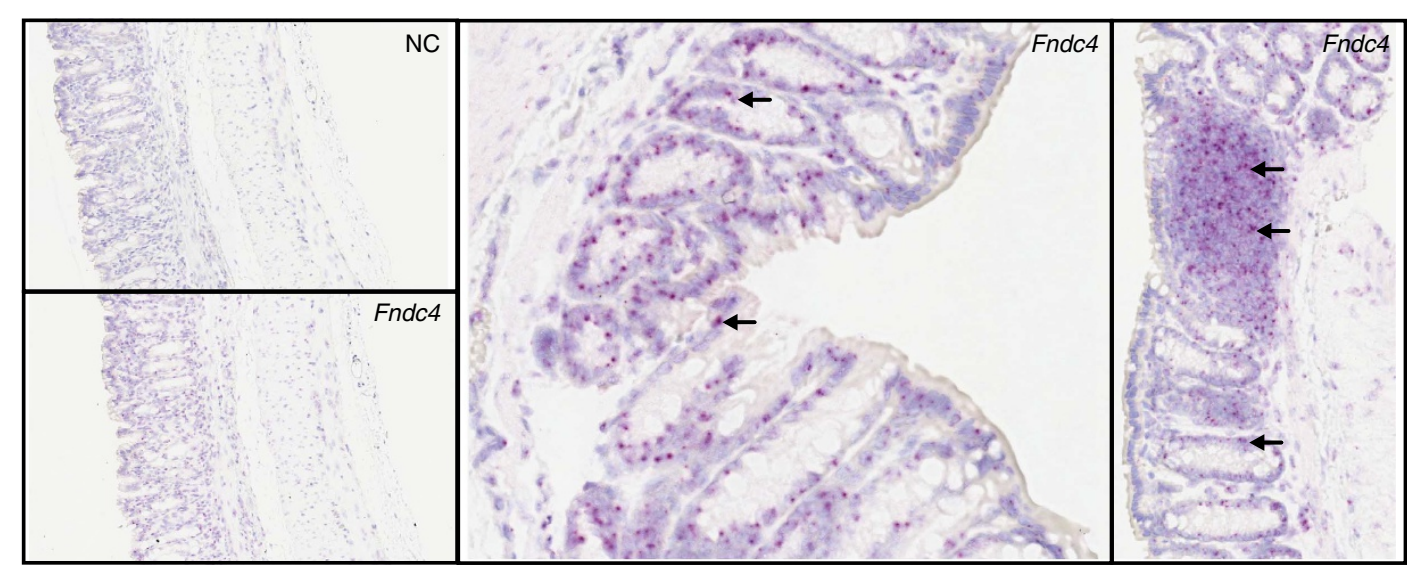

Figure 2 | Fndc4 gene expression is upregulated in inflammatory conditions. (a) Fndc4 gene expression in the murine colon on induction of DSSmediated colitis. DSS was administered via the drinking water, mice were sacrificed 7 days later, $N=10$ mice per group. NT, non-treated. Mean \pm s.e.m., ${ }^{\star} P<0.05$ (Student's $T$-test). This experiment was performed three times with similar results. (b) Fndc4 gene expression in the kidney on induction of glomerulonephritis. Mice were injected with antiserum directed against the glomerular basement membrane ( $\alpha$ GBM) and sacrificed 5 days post injection. NT, non-treated. $N=5$ mice per group. Mean \pm s.e.m., ${ }^{\star} P<0.05$ (Student's $T$-test). This experiment was repeated once with similar results. (c-d) Human IBD cohort. FNDC4 gene expression in intestinal biopsies from inflamed versus non-inflamed regions of the same patients with ulcerative colitis (UC) $(N=12)(\mathbf{c})$ or Crohn's disease $(C D)(N=7)(\mathbf{d}) .{ }^{\star} P<0.05$, paired T-tests. (e) RNA in situ hybridization. Distal colon from DSS-treated mice. Fndc4 versus negative control (NC) probe. Arrows point at examples of positive signals (red).

$T$-test, and $P=0.507$; one-way analysis of variance, respectively, Supplementary Fig. 3a,b). No differences were seen in FNDC4 expression between non-IBD controls and non-inflamed samples from IBD patients $(P=0.370$; $T$-test, Supplementary Fig. $3 \mathrm{c})$. Yet interestingly, FNDC4 expression was significantly increased in inflamed regions of the intestine when compared with non-inflamed regions from the same subjects in both ulcerative colitis $(P=0.002$; paired T-test, Fig. 2c) and Crohn's disease $(P=0.007$; paired $T$-test, Fig. $2 \mathrm{~d})$. Furthermore, among inflamed samples from IBD patients, FNDC4 gene expression correlated with TNF $\quad(r=0.654, \quad P<0.0001$; Pearson's correlation coefficient; Supplementary Fig. 3d) and CCL2 (MCP1) gene expression $(r=0.612, P<0.0001$; Pearson's correlation coefficient; Supplementary Fig. 3e), further supporting the notion that FNDC4 is regulated with inflammation.

Fndc4 is expressed in colonic epithelium and immune cells. To identify the cellular sources of Fndc4 in the inflamed state, we applied RNA in situ hybridization using the high-sensitivity RNAscope technology 9 . Signal analysis revealed that in the murine colon, Fndc4 mRNA is expressed in the epithelium and in a subset of immune cells in lymphoid aggregates (Fig. 2e, right panel). We further investigated Fndc4 gene expression in different immune cell types using publicly available data sets ${ }^{10}$ (Supplementary Fig. 4). This revealed that various human and mouse immune cell types showed low but detectable FNDC4 gene expression (Supplementary Fig. 4a-b). FNDC4 expression tended to be increased in activated human neutrophils (Supplementary Fig. 4a). In mice, natural killer cells and dendritic cells showed the highest levels of Fndc4 expression (Supplementary Fig. 4b). Interestingly, human monocytes showed higher FNDC4 expression levels compared with macrophages (Supplementary Fig. 4c). Yet, expression in immune cells is generally low compared with colonic epithelial cells. In macrophages, Fndc4 gene expression is close to the detection limit with RT-qPCR, whereas Caco-2 colonic epithelial cells show expression levels that are several orders of magnitude higher (Supplementary Fig. 4d). Thus, Fndc4 is expressed in colonic epithelial cells and several immune cell subtypes.

Fndc4 expression is regulated by TGF- $\boldsymbol{\beta}$. Given the bidirectional regulation of Fndc4 and Fndc5 in the models for inflammatory disease and the recent data of Tiano et al. ${ }^{11}$ demonstrating downregulation of Fndc5 by transforming growth factor- $\beta$ (TGF- $\beta$ ), we set out to investigate a potential regulation of Fndc4 by TGF- $\beta$. Interestingly, TGF- $\beta$ treatment induced Fndc4 expression levels in Caco-2 human epithelial cells (Fig. 3a). Furthermore, analysis of the activity of the Fndc4 promoter region using a luciferase readout confirmed TGF- $\beta$-mediated activation, which was blunted by the TGF- $\beta 1 \mathrm{R}$ inhibitor SB-431542 (Fig. 3b). Tumour necrosis factor- $\alpha$ (TNF $\alpha$ ) and lipopolysaccharide (LPS) did not induce Fndc4 expression 


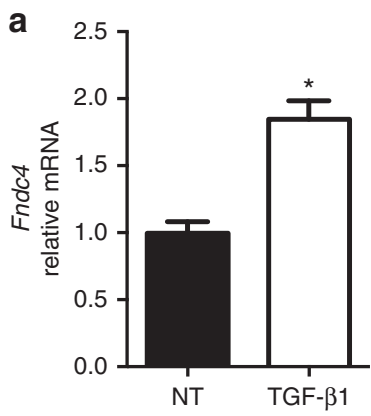

b

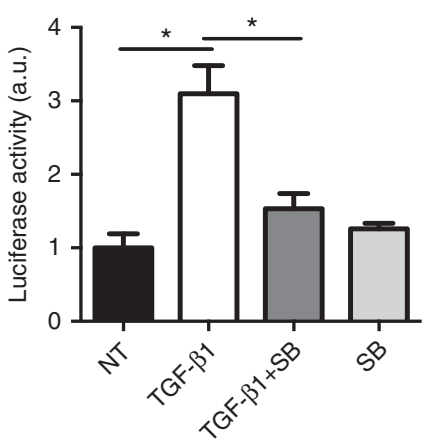

Figure 3 | TGF $\beta$ induces FNDC4 expression. (a) FNDC4 expression in Caco-2 cells after $20 \mathrm{~h}$ treatment with $20 \mathrm{ng} \mathrm{ml}^{-1} \mathrm{TGF} \beta 1 . \mathrm{N}=4$. Mean \pm s.e.m., ${ }^{\star} P<0.05$, Student's $T$-test. (b) Luciferase assay. McA-RH7777 cells were transfected with an Fndc 4 promoter reporter construct and treated with $20 \mathrm{ng} \mathrm{ml}^{-1}$ TGF $\beta 1$ and/or TGF $\beta 1 \mathrm{R}$ antagonist SB-431542 (SB) $(20 \mu \mathrm{M})$ for $20 \mathrm{~h} . \quad N=4-5$. Mean \pm s.e.m., ${ }^{\star} P<0.05$ (one-way analysis of variance with Tukey's post hoc tests).

(Supplementary Fig. 4e-f). Thus, our data suggest that there are several potential explanations for increased Fndc4 expression during inflammation, including TGF- $\beta$-induced expression in epithelial cells and increased infiltration of Fndc4 expressing immune cells.

FNDC4 reduces colitis disease progression in mice. We next sought to investigate the functional consequences of increased Fndc4 expression in inflammation using a gain-of-function system in a rodent model. To this end, we produced FNDC4 (extracellular domain only) conjugated to the $\mathrm{Fc}$-domain of human IgG1 $(\mathrm{hFc})$ resulting in a biophysically stable protein with good kinetic properties (Supplementary Fig. 5). Importantly, all experiments were controlled with an isotype matched $\mathrm{hFc}$ control. Mice were injected with $\mathrm{hFc}$-FNDC4 or $\mathrm{hFc}$ control followed by induction of colitis by addition of DSS to the drinking water for 5 days. Injections were repeated on days 2 and 4 and mice were sacrificed on day 6. Interestingly, in the FNDC4-treated group, colitis severity was significantly ameliorated, as demonstrated by a lower disease activity index (DAI) ${ }^{12}$ score (Fig. 4a), and a reduced shortening of the colon length, a surrogate marker for the degree of inflammation in the DSS model (Fig. 4b). Consistently, the FNDC4-injected mice lost significantly less weight, indicating a milder disease course (Fig. 4c). We also analysed gene expression in intestinal samples from these mice, where a robust induction of proinflammatory genes was observed on DSS treatment. Many of these genes were reduced in the FNDC4-treated mice compared with the control cohort (Fig. 4d,e). Especially Cxcl9, Cxcl10 (Ip10), Tgfb1 and Csf1 were significantly reduced in the FNDC4-treated group. Furthermore, histopathology scores for colitis severity were significantly lower in the $\mathrm{hFc}$-FNDC4-treated mice (Fig. 4f), supporting the beneficial effects of $\mathrm{hFc}$-FNDC4 supplementation in colitis. There were no significant effects of hFc-FNDC4 treatment on body weight and colonic inflammatory gene expression in healthy control mice (Supplementary Fig. 6). In summary, therapeutic delivery of FNDC4 reduced the disease severity of colitis in mice.

FNDC4 acts on macrophages. Subsequently, we sought to elucidate the cell type targeted by FNDC4 in colitis. To this end, we first analysed the composition of the immune cell population in the colons of DSS-treated mice using FACS and found that the majority of the CD45-positive cells were positive for $\mathrm{CD} 11 \mathrm{~b}$
(Fig. 5a). CD11b is expressed by macrophages, neutrophils and eosinophils, consistent with their reported predominance in inflammatory infiltrates in DSS-induced colitis ${ }^{13}$. We next used a FACS-ligand-binding assay to study the binding of hFc-FNDC4 to different immune cells. To this end, we utilized Fc-gamma receptor-deficient mice ( $F c g / 2 b$ knockout $(\mathrm{KO})$; a cross of the mouse strains described in refs 14,15) to completely exclude any minor binding mediated by the $\mathrm{hFc}$ part of the $\mathrm{hFc}$-FNDC4 fusion protein. Interestingly, FNDC4 displayed strong, specific binding to macrophages/monocytes, whereas neutrophils, eosinophils, $\mathrm{T}$ cells and NK cells did not display specific binding (Fig. 5b). Moreover, bone marrow-derived macrophages treated with FNDC4 in vitro displayed ERK phosphorylation (Fig. 5c) and dose-dependent target gene activation (these genes were consistently regulated on FNDC4 treatment in a range of different cell types and are used as readout for bioactivity of the $\mathrm{hFc}$-FNDC4 recombinant protein; Fig. 5d) as expected ${ }^{16}$, demonstrating that FNDC4 induces active signalling in macrophages. As resident and recruited macrophages constitute a major cell type in acute colitis and ablation of macrophages exerts a beneficial effect on acute intestinal inflammation ${ }^{17}$, our results suggest that the inhibitory effects of FNDC4 on macrophages are of central importance for its therapeutic effects in the DSS model.

FNDC4 treatment reduces macrophage phagocytosis. A series of assays were used to assess the consequences of FNDC4 stimulation of bone marrow-derived macrophages in vitro. Strikingly, FNDC4 treatment strongly reduced phagocytosis of fluorescent beads (Fig. 6a). Consistent results were obtained for dead cell clearance (phagocytosis of apoptotic thymocytes; Supplementary Fig. 7a). Moreover, FNDC4-enhanced survival of macrophages after serum depletion (Fig. 6b), without indications for enhanced macrophage proliferation (Supplementary Fig. 7b). Finally, TNF $\alpha$-induced production of nitric oxide (NO) production was blunted (Supplementary Fig. 7c). Thus, FNDC4 treatment resulted in a state of reduced macrophage activity, but enhanced survival. To address the transcriptional response to FNDC4 stimulation, we performed whole-genome gene expression analysis (significantly regulated genes with a fold change above 1.5 are listed in Supplementary Table 3), which confirmed the downregulation of many key pathways for macrophage activation (Supplementary Table 4). This gene ontology analysis in particular highlighted effects on phagocytosis, survival (phosphatidylinositol 3-kinase cascade), chemotaxis and cytokine secretion. Using RT-qPCR, we validated many of these findings with the reduction in the expression of key phagocytosis genes (Supplementary Fig. 8a), but also chemokines and cytokines (Fig. 6c). Importantly, this suppression of proinflammatory genes was observed both in the presence and the absence of TNF $\alpha$ as inflammation trigger (Fig. 6d) and was also observed in peritoneal macrophages treated with hFc-FNDC4 (Supplementary Fig. 8b). Thus, FNDC4 treatment results in an anti-inflammatory, quiescent-state macrophage as judged from activity assays and gene expression.

These effects could possibly have been attributed to 'M2' polarization of the macrophages in culture ${ }^{18}$, but as shown in Fig. 6e,f, this was not the case. Interestingly, both classical M1 (Ccl2, Cxcl9, Cxcl10, Fig. 6c,d) and M2 markers (Mrc1, Clec10a, Fig. 6f) were downregulated by FNDC4 in the basal state. The M1 markers Tnf and Nos2 (Fig. 6e) and the M2 marker Tgfb1 (Fig. 6f) were unchanged after hFc-FNDC4 treatment in the basal state. Likewise, hFc-FNDC4 treatment resulted in downregulation of M1 and M2 markers after LPS- or interleukin-4 (IL-4)-mediated polarization towards M1 or M2 phenotypes, respectively 


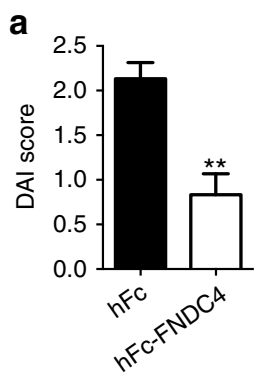

e

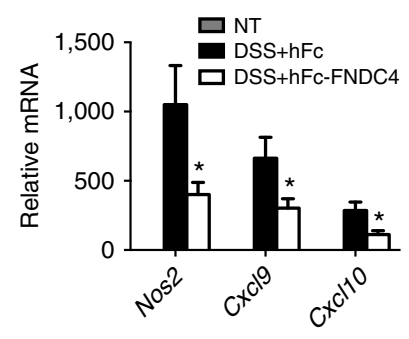

b

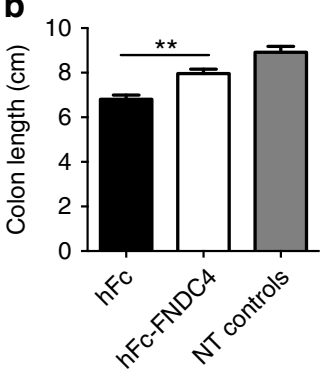

f

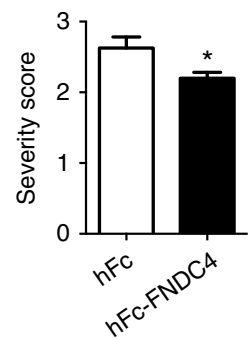

C

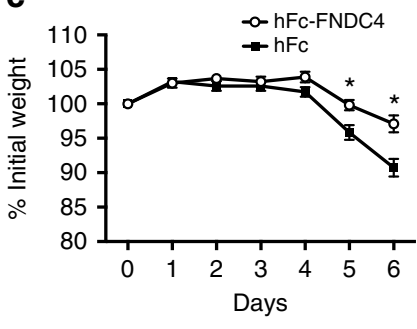

NT d

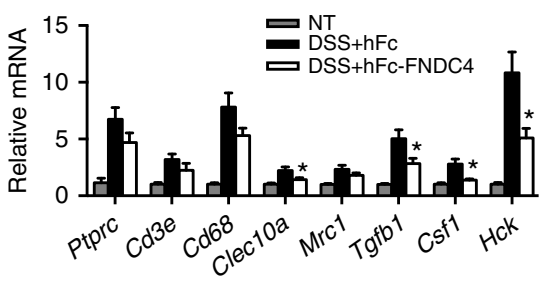

DSS+hFc

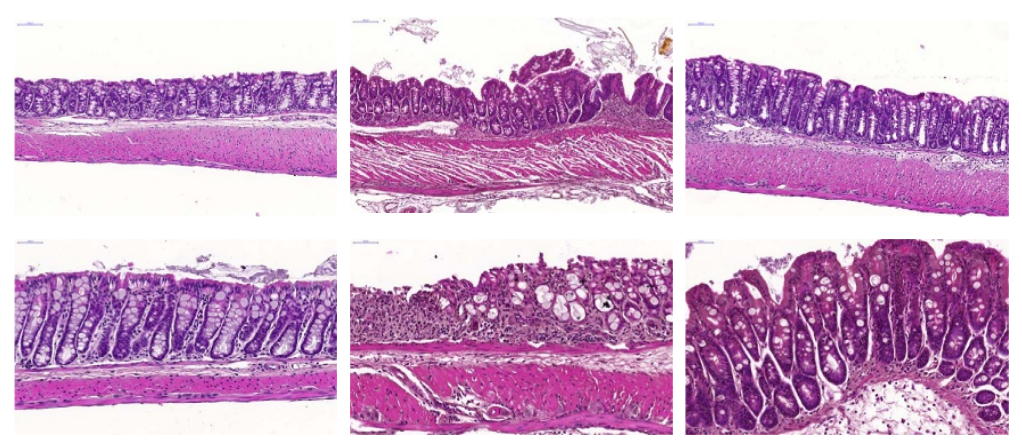

DSS+hFc-FNDC4

Figure 4 | FNDC4 improves colitis in mice. DSS-induced colitis model. Mice were administered DSS from day 0 to 5 and were injected with hFc-FNDC4 or hFc control protein on days 0,2 and $4 . N=10$ mice per group. This experiment was performed three times (of which the last experiment was blinded, blinding was performed by a colleague not involved in the study) with similar results. (a) DAI (disease activity index). ${ }^{\star \star} P<0.001$, Mann-Whitney rank test. (b) Colon length. ${ }^{\star \star} P<0.001$, Student's $T$-test. The non-treated (NT) control group consisted of 5 age- and gender-matched controls that did not receive DSS. (c) Body weight curve. ${ }^{\star} P<0.05$, Student's T-test. (d-e) Gene expression, selected genes. Mean \pm s.e.m., ${ }^{\star} P<0.05$ and $q<0.10$; two-sided Student's T-tests of hFc versus hFc-FNDC4, followed by the Benjamini-Hochberg false discovery rate (FDR) correction for multiple testing. (f) Histopathology. Colitis severity assessment and representative images. Scores are mean \pm s.e.m. ${ }^{\star} P<0.05$, Mann-Whitney rank test.

(Fig. 6g,h), whereas identity markers such as Ptprc (CD45) and Emr1 (F4/80) were unchanged (Fig. 6i). Thus, without signs of de-differentiation, these cells become largely quiescent after stimulation with FNDC4.

FNDC4 also acts on primary human macrophages. Excluding the signal peptide, the ectodomain of human FNDC4 is $100 \%$ homologous to mouse FNDC4. Thus, hFc-FNDC4 is suitable for both mouse and human applications. We therefore examined the potential effects of FNDC4 on primary human macrophages from four different donors. hFc-FNDC4 treatment significantly downregulated TNF and CCL2 (MCP1) expression in both the basal (Fig. 7a) and LPS-stimulated state (Fig. 7b). Furthermore, under LPS-stimulated conditions, $\mathrm{hFc}$-FNDC4 treatment also reduced the expression of the proinflammatory chemokines CCL3, CCL4 and CXCL9 (Fig. 7b).

FNDC4 KO mice exposed to DSS show increased colitis severity. To evaluate whether FNDC4 is not only sufficient, but also required for immune dampening in colitis, we investigated DSS-induced colitis development and severity in Fndc4 KO mice. Fndc4 KO mice were generated by crossing mice with floxed Fndc4 alleles with CMV-Cre mice. Heterozygote breeding pairs gave normal Mendelian distribution and average litter sizes (average litter size of seven mice). Furthermore, the KO mice had no detectable developmental defects. Body weight was similar between Fndc4 KO and littermate controls (Fig. 8a). Furthermore, spleen appearance (histology) and weight (Fig. 8b) was unaffected. Moreover, baseline gene expression profiles of a panel of immune cell and inflammation markers were similar in spleens of KO and WT mice (Supplementary Fig. 9).
Induction of colitis resulted in increased disease severity in the Fndc4 KO mice, as shown by increased disease severity index (Fig. 8c), reduced colon length (Fig. 8d), increased body weight loss (Fig. 8e), increased histopathology scores (Fig. 8f,g) and increased proinflammatory gene expression (Fig. 8h).

FNDC4 acts partly via STAT3. We then proceeded to identify signalling pathways involved in the effects of FNDC4 on macrophages. STAT3 is an important regulator of inflammatory processes ${ }^{19,20}$ and was shown to be activated on irisin treatment of neuronal cells ${ }^{21}$. Furthermore, gene expression analysis of bone marrow-derived macrophages treated with hFc-FNDC4 revealed overrepresentation of the GO classes associated with peptidyl-tyrosine phosphorylation and JAK-STAT signalling (Supplementary Table 3). Therefore, we examined whether FNDC4 affects STAT3 activation. The STAT3 target gene Socs3 was increased fourfold after $4 \mathrm{~h}$ of treatment with $100 \mathrm{nM} \mathrm{hFc}$ FNDC4 protein (Fig. 9a) and showed a dose-response pattern (Supplementary Fig. 10a). Furthermore, phosphorylation of STAT3 (Fig. 9b; but not STAT6, Supplementary Fig. 10b) was induced after $30 \mathrm{~min}$ of FNDC4 treatment. FNDC4-mediated STAT3 activation was further supported by STAT3 DNA binding to the Socs3 promoter region (chromatin immunoprecipitation (ChIP) assay; Fig. 9c). Importantly, inhibition of STAT3 resulted in reversal of gene regulation of several FNDC4 regulated genes (Fig. 9d) and reversal of FNDC4-mediated improvements in macrophage survival (Fig. 9e), indicating that STAT3 is at least partly responsible for FNDC4 downstream signalling.

To identify other pathways involved in the actions of FNDC4 on macrophages, we performed a phospho-antibody array containing 1,318 site-specific antibodies from over 30 signalling pathways. The phosphorylation sites with a foldchange of at least 

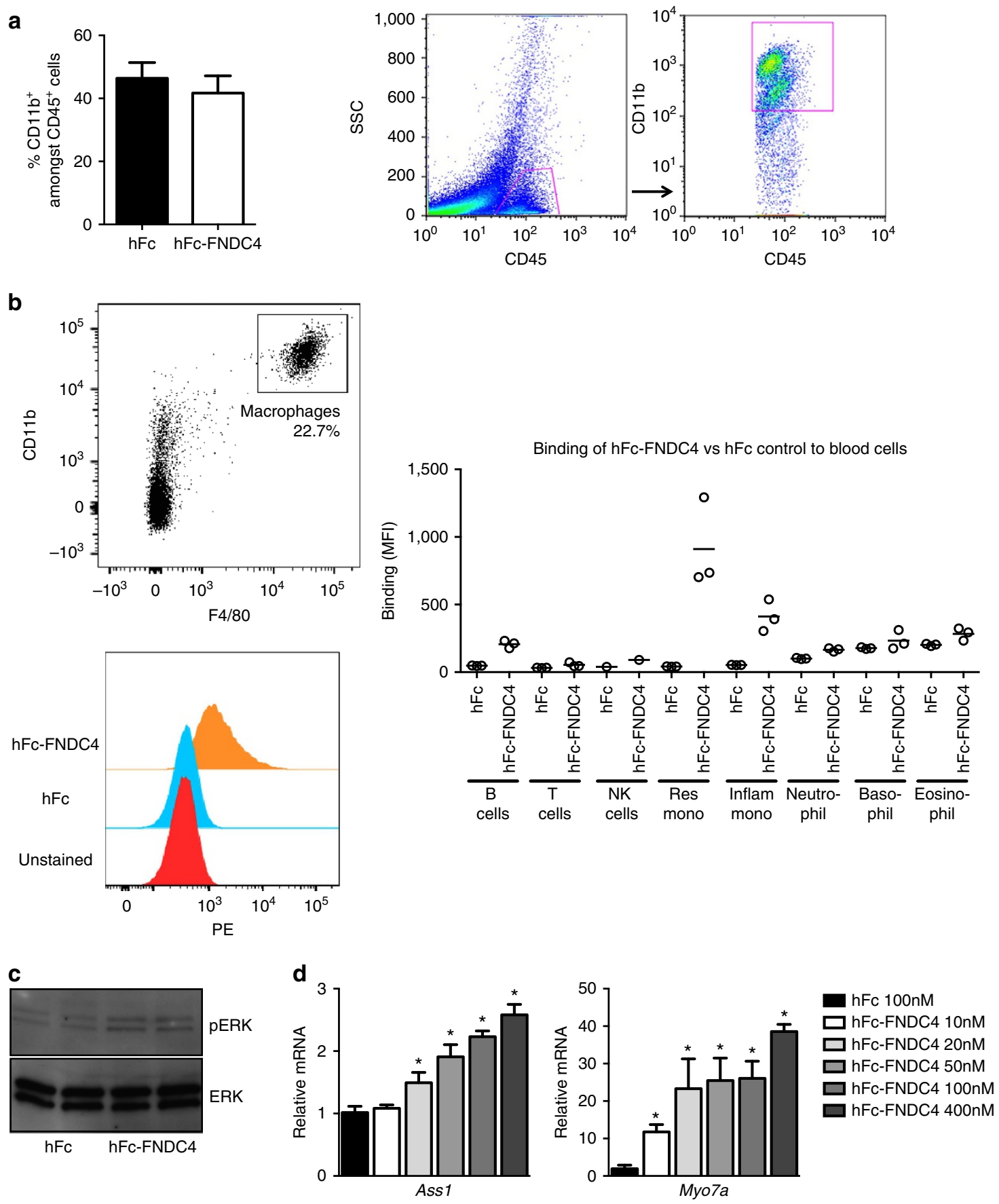

Figure 5 | FNDC4 acts on macrophages. (a) Immune cell infiltration of DSS-mediated inflamed colon mostly consists of macrophages. Flow cytometry analysis of $\mathrm{CD}_{11 \mathrm{~b}}{ }^{+}$-cells amongst $\mathrm{CD} 45^{+}$cells in the DSS colitis model. $N=10$ for the hFc control group, $N=9$ for the hFc-FNDC4 group. (b) FNDC4 binds to IgG Fc-gamma (IgG Fc-receptor negative) macrophages. The plots on the left show binding to peritoneal macrophages, the graph on the right shows binding to a panel of blood cells. Antibodies against CD11b and F4/80 were used to gate for macrophages. Binding of hFc-FNDC4 to cells was detected using a PE-conjugated antibody against human IgG. (c) ERK phosphorylation after 30 minutes of hFc-FNDC4 treatment. Total ERK served as loading control. This experiment was repeated twice with similar results. The full-size blot image is presented in Supplementary Fig. 11b. (d) Dose curve of hFc-FNDC4-mediated induction of Ass1 and Myo7a in bone marrow macrophages treated for $24 \mathrm{~h}$ with different concentrations of hFc-FNDC4 (100 nM hFc as control). Data are presented as mean \pm s.e.m., $N=4$. ${ }^{\star} P<0.05$, One-way analysis of variance followed by Dunnett's post hoc tests.

1.3 are listed in Fig. 9f. Phosphorylation of FER (pTyr402) and HSP90B (pSer226) was reduced by FNDC4 treatment, while LYN (pTyr507) and MAPKAPK2 (pThr334) showed the strongest reduction in phosphorylation ratio due to increased total protein binding, indicating possible conformational changes in the protein. FNDC4-induced phosphorylation was strongest for
Connexin 43 (pSer367), Cyclin D1 (Thr286) and NFкB p105/p50 (pSer337). Abl1 (pTyr204), Calmodulin (pThr79/pSer81) and Caspase 9 (pTyr153) showed increased phosphorylation ratios due to reduced total protein binding.

In summary, we have characterized a novel secreted factor-FNDC4-which signals to macrophages to downregulate 
a

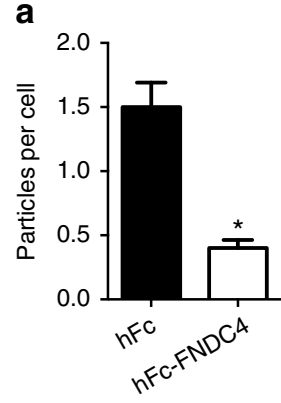

c

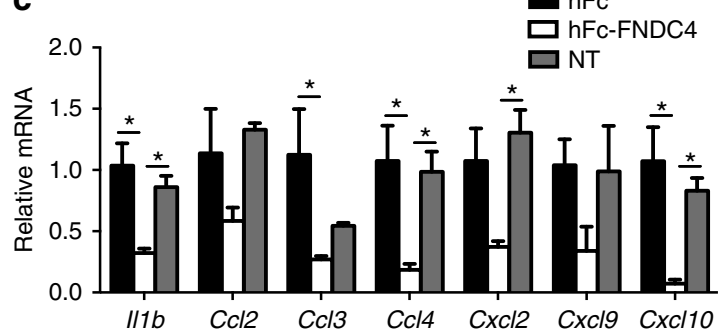

e

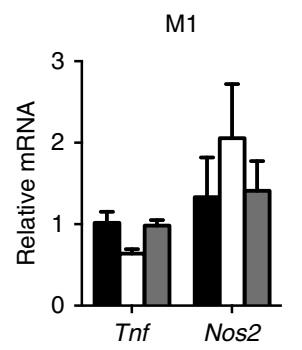

g

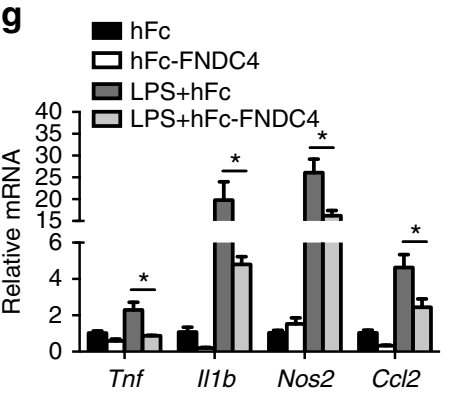

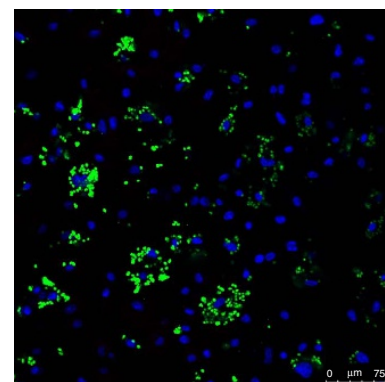

$\mathrm{hFc}$

$\mathrm{hFc}$

口Ff-FNDC4 $\square \mathrm{N}$

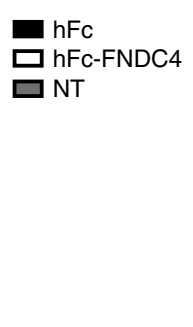

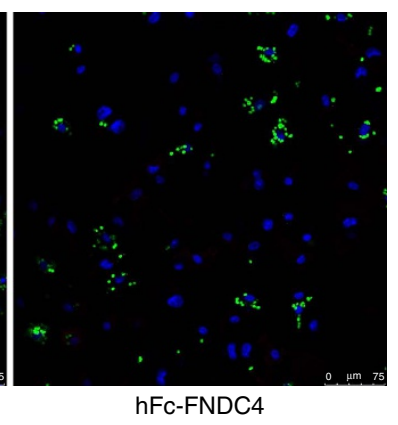

b

d

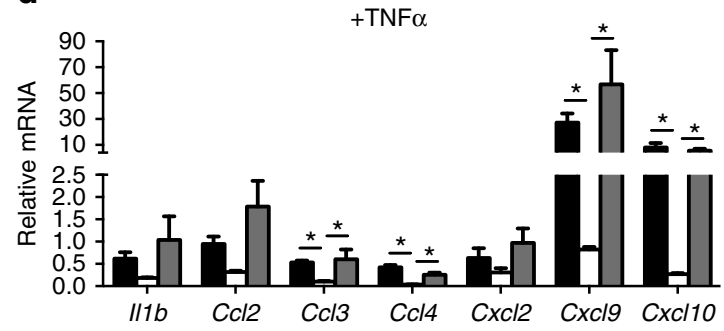

f

M2

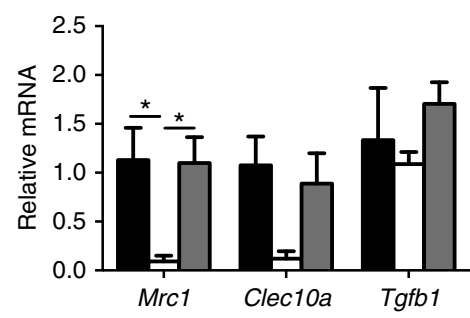

i

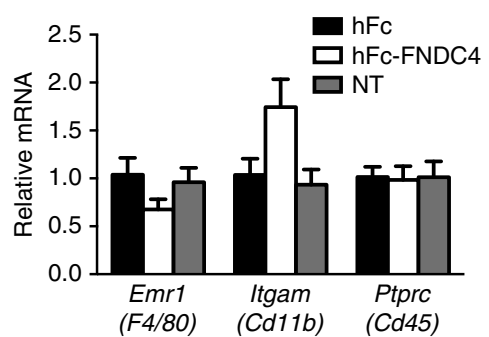

Figure 6 | FNDC4 treatment affects key macrophage processes and downregulates proinflammatory gene expression. (a) Phagocytosis of fluorescently labelled zymosan A particles. Bone marrow macrophages were treated for $24 \mathrm{~h}$ with $100 \mathrm{nM} \mathrm{hFc-FNDC4}$ or hFc control followed by incubation with zymosan $A$ particles for 30 minutes. Mean \pm s.e.m., $N=4$. ${ }^{\star} P<0.05$ ( $T$-test). This experiment was performed twice with similar results. (b) Survival of bone marrow-derived macrophages after $48 \mathrm{~h}$ in starvation media, treated with $100 \mathrm{nM} \mathrm{hFc}$-FNDC 4 or hFc control. Mean \pm s.e.m., $N=4$. ${ }^{\star} P<0.05$ ( $T$-test). This effect was validated in two individual repeat experiments. (c) Gene expression of a selection of cytokines and chemokines on hFc-FNDC4 treatment (or hFc as carrier-control) (100 nM). N=6-7 (d) Gene expression of a selection of cytokines and chemokines on hFc-FNDC4 treatment (24h, $100 \mathrm{nM}$ ) in the presence of TNF $\alpha . N=6-7$. (e-f) Gene expression of M1 (e) and M2 (f) markers. N=6-7. (g) Gene expression of Tnf, II7b and Nos2 after LPS-mediated polarization. Cells were treated with $10 \mathrm{ng} \mathrm{ml}{ }^{-1}$ LPS for 3 days before $24 \mathrm{~h}$ treatment with $100 \mathrm{nM} \mathrm{hFc-FNDC} 4$ or hFc control. $N=4$. (h) Gene expression of $\mathrm{M} 2$ markers Clec10a and Mrc1 after IL-4-mediated polarization. Cells were treated with $10 \mathrm{ng} \mathrm{ml}-1 \mathrm{IL}-4$ for 3 days before $24 \mathrm{~h}$ treatment with $100 \mathrm{nM}$ hFc-FNDC4 or hFc control. $N=4$. (i) Gene expression of the general leukocyte marker Ptprc (Cd45) and the general macrophage markers Emr1 (F4/80) and Itgam (Cd11b). $N=3$. Mean \pm s.e.m. NT, non-treated. ${ }^{\star} P<0.05$ and $q<0.10$ for the comparison hFc-FNDC 4 versus the corresponding hFc control group; one-way analysis of variance with Tukey's post hoc tests, followed by the Benjamini-Hochberg FDR correction. These genes were regulated to a similar extend in the gene expression array and similar effects on gene expression were seen in multiple $(>3)$ independent validation experiments.

inflammation. FNDC4 supplementation reduced disease severity in a mouse model for colitis, indicating therapeutic potential.

\section{Discussion}

Here we characterized the immunomodulating properties of FNDC4, of which to our knowledge no functional reports exist to date. FNDC4 treatment of mice with DSS-induced colitis resulted in reduced inflammation and clinical improvement, suggesting therapeutic potential. We show that FNDC4 binds to and acts on macrophages to downregulate proinflammatory gene expression. Furthermore, key macrophage functions including phagocytosis were strongly affected by FNDC4 treatment, and these effects 

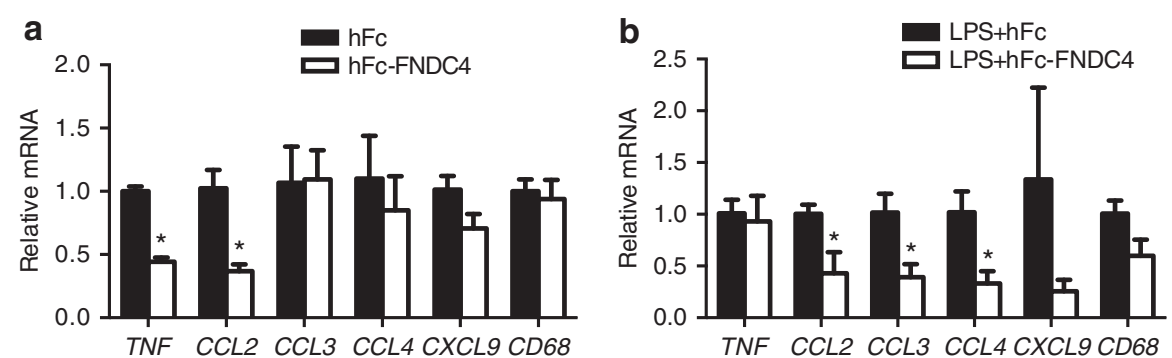

Figure 7 | FNDC4 also acts on human macrophages. Effects of hFc-FNDC4 treatment on proinflammatory gene expression in the basal (3-4 individual donors) (a) and LPS-stimulated state (2-3 individual donors) (b). Macrophages were cultured in the presence of macrophage colony-stimulating factor and treated with hFc-FNDC4 or hFc control for $24 \mathrm{~h}$. Subsequently, RNA was isolated using trizol, reverse transcribed and subjected to RT-qPCR analysis. Mean \pm s.e.m., ${ }^{\star} P<0.05$ (T-test).
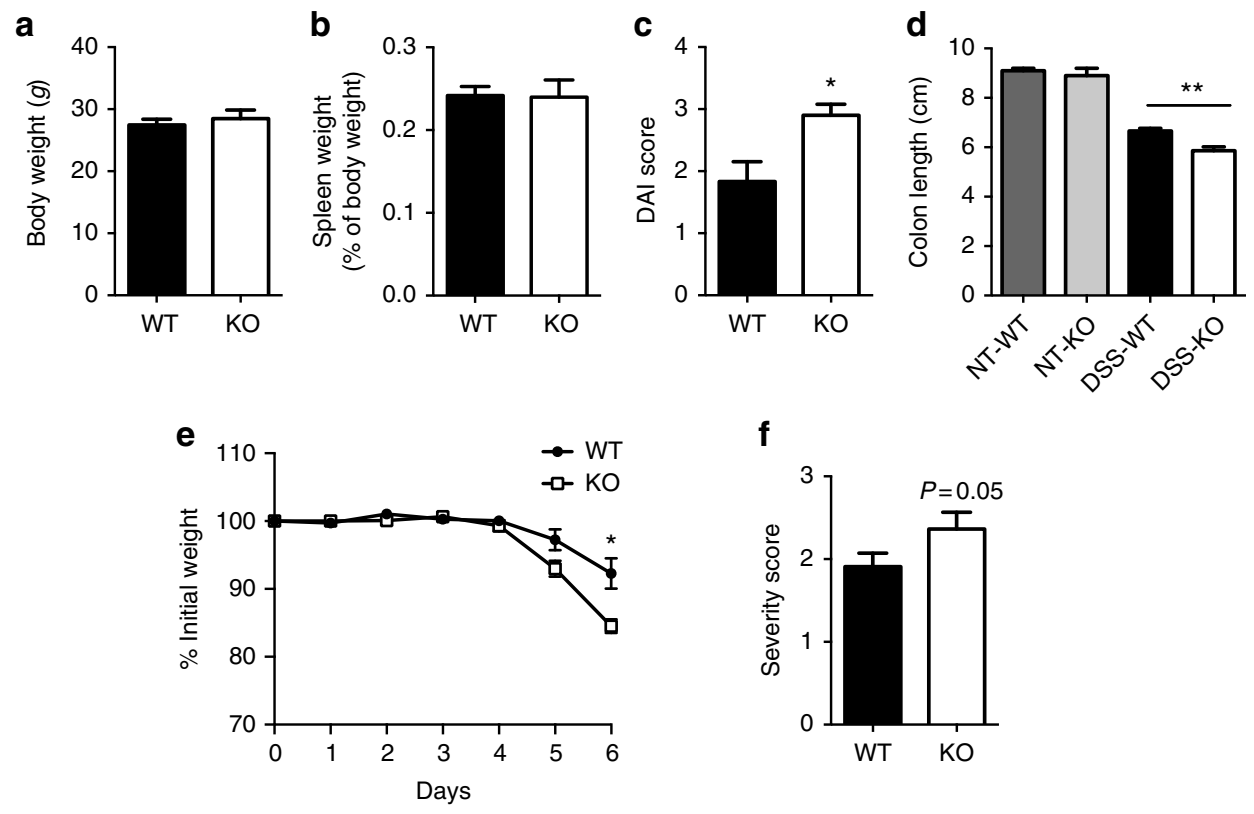

f

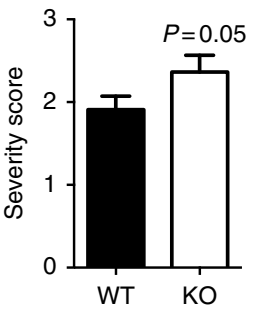

g

WT

$\mathrm{KO}$
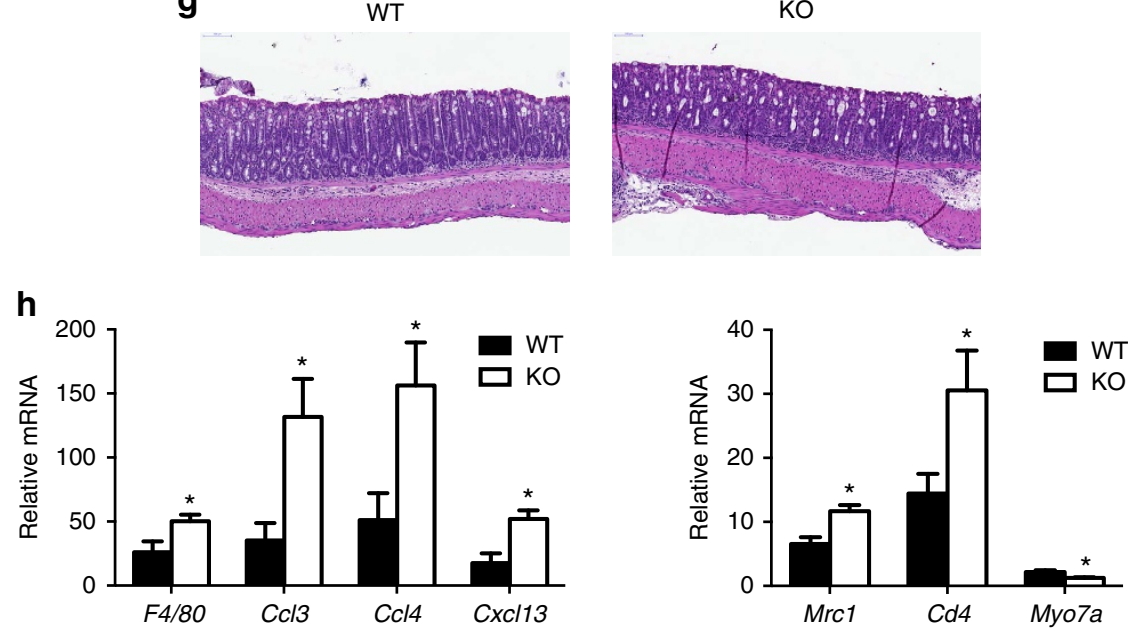

Figure 8 | FNDC4 knockout mice show increased inflammation and severity of DSS-induced colitis. (a) Body weights of 12 week old mice, $N=4$. (b) Spleen weight, standardized to body weight at 16 weeks of age, $N=4$. (c) DAl (disease activity index). $N=5$ mice per group. ${ }^{\star} P<0.05, M a n n-W h i t n e y$ rank test. KO, Fndc4 knockout mice, WT, wild-type mice. (d) Colon length. ${ }^{\star} * P<0.01$, analysis of variance with Tukey's post hoc test. (e) Body weight curve. ${ }^{\star} P<0.05$ and $q<0.05$, Student's T-test followed by the Benjamini-Hochberg false discovery rate correction. (f-g) Histopathology. Colitis severity assessment. $N=11$ mice per group, ${ }^{\star} P<0.05$, Mann-Whitney rank test. (h) Gene expression, selected genes. ${ }^{\star} P<0.05$; two-sided Student's $T$-tests, $N=5$ mice per group. Disease severity was assessed blinded and the experiment was repeated with similar results. Mean \pm s.e.m. 
a

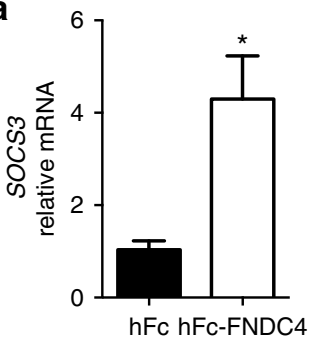

C

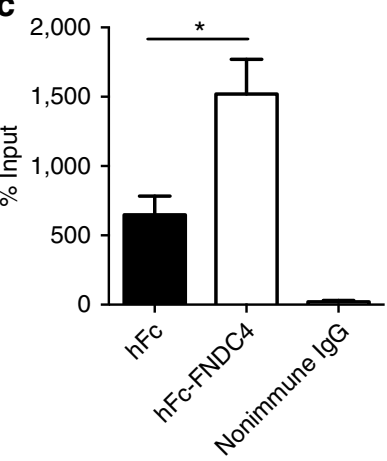

e

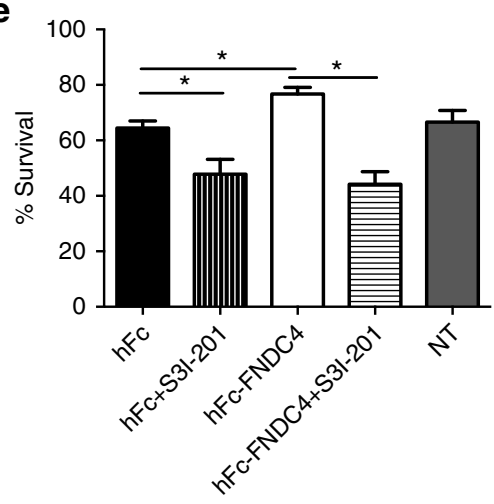

b
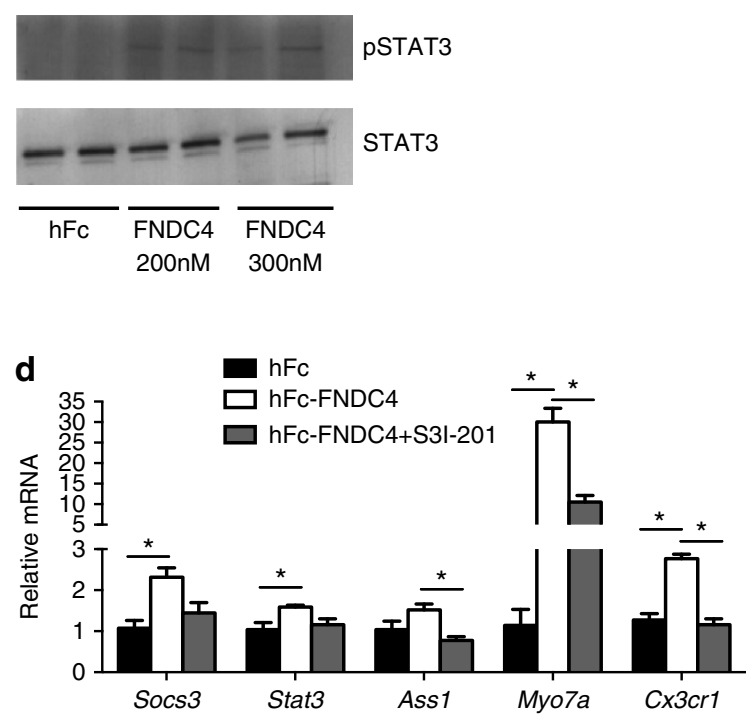

\begin{tabular}{ll}
\hline f & Ratio \\
\hline Abl1 (Phospho-Tyr204) & 2,21 \\
Calmodulin (Phospho-Thr79/Ser81) & 2,03 \\
Connexin 43 (Phospho-Ser367) & 1,70 \\
Cyclin D1 (Phospho-Thr286) & 1,61 \\
Caspase 9 (Phospho-Tyr153) & 1,49 \\
NFkB-p105/p50 (Phospho-Ser337) & 1,47 \\
MSK1 (Phospho-Ser360) & 1,46 \\
MKK3 (Phospho-Ser189) & 1,41 \\
SHP-2 (Phospho-Tyr580) & 1,38 \\
GATA1 (Phospho-Ser142) & 1,38 \\
4E-BP1 (Phospho-Ser65) & 1,37 \\
CDC2 (Phospho-Tyr15) & 1,36 \\
Estrogen Receptor-alpha (Phospho-Ser104) & 1,36 \\
Raf1 (Phospho-Tyr341) & 1,33 \\
PLCG2 (Phospho-Tyr753) & 1,31 \\
Smad3 (Phospho-Ser425) & $*$ \\
CASP6 (Phospho-Ser257) & 1,31 \\
Filamin A (Phospho-Ser2152) & 1,31 \\
P9ORSK (Phospho-Thr359/Ser363) & 1,30 \\
TOP2A/DNA topoisomerase II (Phospho-Ser1106) & 1,30 \\
p53 (Phospho-Ser315) & 0,77 \\
FER (Phospho-Tyr402) & 0,76 \\
LYN (Phospho-Tyr507) & 0,68 \\
HSP90B (Phospho-Ser226) & * \\
MAPKAPK2 (Phospho-Thr334) & 0,63 \\
\hline
\end{tabular}

Figure 9 | FNDC4 signals partly via STAT3. (a) Socs3 gene expression after $4 \mathrm{~h}$ incubation with $100 \mathrm{nM} \mathrm{hFc-FNDC4} \mathrm{or} \mathrm{hFc} \mathrm{control.} \mathrm{Mean} \pm$ s.e.m., $N=3$. ${ }^{\star} P<0.05$ (T-test). (b) Phosphorylation of STAT3. Bone marrow-derived macrophages were starved overnight followed by 30 minutes treatment with $200 \mathrm{nM}$ or $300 \mathrm{nM}$ hFc-FNDC4, $200 \mathrm{nM}$ hFc served as control. The full-size, uncropped blot image is presented in Supplementary Fig. 11c. Similar effects were observed in two repeated experiments. (c) STAT3 DNA binding to the Socs3 promoter region. Macrophages were treated with hFc-FNDC4 or hFc for $6 \mathrm{~h}$. Data presented as percentage of input. Mean \pm s.e.m., $N=4$. ${ }^{\star} P<0.05$ ( $T$-test). (d) STAT3 inhibition reverses FNDC4-mediated gene regulation. Bone marrow-derived macrophages were pretreated with $50 \mu \mathrm{M}$ S3I-201 STAT3 inhibitor or DMSO as control for 45 min and subsequently treated with $100 \mathrm{nM}$ $\mathrm{hFc}-\mathrm{FNDC4}$ or $\mathrm{hFc}$ control for $6 \mathrm{~h}$ in the presence or absence of S3I-201. Mean \pm s.e.m., ${ }^{\star} P<0.05$ and $q<0.10$, One-way analysis of variance followed by Tukey's post hoc tests and Benjamini-Hochberg false discovery rate-adjustment, $N=4$. (e) STAT3 inhibition reverses FNDC4-mediated improvements of macrophage survival. Bone marrow macrophages were pretreated with $50 \mu \mathrm{M}$ S3I-201 STAT3 inhibitor or DMSO as control for 45 min and subsequently treated with $100 \mathrm{nM} \mathrm{hFc-FNDC4}$ or hFc control for $48 \mathrm{~h}$ in starvation media in the presence or absence of S3I-201. NT, non-treated. Mean \pm s.e.m., ${ }^{\star} P<0.05$, one-way analysis of variance followed by Tukey's post hoc tests, $N=8$ per condition. (f) Proteins showing a $>1.3$-fold increase or decrease in phosphorylation status in primary bone marrow macrophages on 30-min treatment with hFc-FNDC4 or hFc control. The fold change was calculated as the signal ratio of the paired antibodies for each pair of site-specific antibody and phospho site-specific antibody. Average ratios of two duplicates per condition. * Indicate that the fold induction in phosphorylation status is due to a reduction in binding of the total protein (conformational change affecting the accessibility of the epitopes; the array is performed using non-denatured protein).

were partly mediated by STAT3 activation. Importantly, Fndc4 KO mice showed increased colitis severity, suggestive of a requirement for FNDC4 to dampen the immunological response in colitis.

Fndc4 gene expression was upregulated with inflammation in human IBD and in inflamed tissue in several mouse models for inflammatory diseases including DSS colitis and nephritis, indicating that FNDC4 may play a physiological role in the regulation of inflammation, potentially counteracting (consequences of) inflammation. Furthermore, we showed that TGF- $\beta 1$ upregulates Fndc4 expression, which might explain the induction of Fndc4 expression with inflammation, in addition to potentially increased infiltration of Fndc4-expressing immune cells. Fndc4 is highly expressed in many tissues including liver, brain, heart, adipose tissue and spleen, while it is barely expressed in macrophages. It is thus likely that non-macrophage cells signal to macrophages in a paracrine- or endocrine fashion. The cellular source of FNDC4 protein in inflammatory disease will be an important focus for 
subsequent studies. The lack of a robust and valid antibody for detection of endogenous FNDC4 levels prevented us from including endogenous protein expression data in the current report. Yet, in situ RNA hybridization experiments revealed FNDC4 expression in colonic epithelium as well as a subset of immune cells in lymphoid aggregates. Immune cell expression profiles further support expression of Fndc4 in several immune cell subtypes, including neutrophils, natural killer cells, dendritic cells and T-cell subtypes. Also monocytes showed Fndc4 expression. However, as for many other peptide hormones, the cellular source for FNDC4 during inflammation is not fully clarified. This will need to be addressed in future studies.

FNDC4 displayed strong binding to macrophages and dampened macrophage inflammatory gene expression. Macrophages were also the most prominent inflammatory cell type in the colitis model used. Moreover, inflammation in colitis is largely mediated by macrophages, as supported by reports demonstrating beneficial effects of targeting macrophage inflammation in mouse models for colitis $22-24$. Altogether, this suggests that FNDC4 exerts its anti-inflammatory effects via macrophages, although we cannot completely rule out contributing effects on other (nonimmune) cell types.

Administering FNDC4 to mice with DSS-induced colitis resulted in downregulation of expression of the proinflammatory chemokines Cxcl9 and Cxcl10 (Ip10), consistent with the in vitro data in macrophages. In accordance, beneficial effects of counteracting chemokine signalling in DSS-induced colitis have been shown in several reports. Tokuyama et al. ${ }^{25}$ showed attenuation of DSS-induced colitis on treatment with a broad spectrum chemokine receptor antagonist. Furthermore, Chami et al. ${ }^{26}$ showed that blockage of $\mathrm{Cxcl} 9$ and $\mathrm{Cxcl} 10$ signalling by loss of function of its receptor, CXCR3, resulted in attenuation of colitis symptoms. Thus, reduced secretion of relevant chemokines by macrophages represents a relevant therapeutic response to FNDC4 treatment.

Interestingly, we observed a strong downregulation of in vitro phagocytosis activity of bone marrow macrophages in culture after treatment with FNDC4. It is thus likely that therapeutic delivery of FNDC4 partially blocks phagocytosis also in vivo, which would be consistent with previous reports where blockage of phagocytosis function attenuated colitis in mice ${ }^{27}$.

Another interesting finding is the downregulation of both M1 and M2 markers on FNDC4 treatment of macrophages in culture. Previously, macrophage activation has been characterized as polarized to either M1 or M2 phenotypes. Yet, recent developments in the field indicate that this classification is too simplified and that there is a spectrum of macrophage functional states spanning between M1 and M2 definitions ${ }^{28,29}$. Our data indicates that FNDC4 action on macrophages results in a quiescent-state macrophage with increased survival but downregulated expression of several M1 and M2 markers. This phenotype did not match any of the previously described M2 subpopulations neither the recently proposed M3 'repair' phenotype ${ }^{30}$. Further research will be required to further characterize this macrophage state.

The effects of FNDC4 on macrophages were partly mediated by STAT3, which is a transcription factor involved in regulating inflammatory processes and cell survival ${ }^{31,32}$. The role of STAT3 in inflammation, however, is complex and cell type specific. For example, targeted deletion of STAT3 in myeloid cells in mice results in spontaneous chronic enterocolitis ${ }^{33}$, while STAT3 deletion in $\mathrm{T}$ cells is associated with reduced colitis symptoms ${ }^{34,35}$. Likewise, risk allele analysis has indicated that both increased and reduced STAT3 activity is associated with increased risk for $\mathrm{IBD}^{36,37}$. Since FNDC4 mainly targets macrophages, STAT3 activation may have contributed to the attenuation of colitis symptoms in the FNDC4-treated mice. Yet, FNDC4 effects were only partly STAT3 dependent. Other transcription factors and downstream signalling pathways are likely to contribute to the anti-inflammatory actions of FNDC4. In addition to the STAT3 target gene Socs3, Socs2 was strongly upregulated in macrophages treated with FNDC4, indicating possible involvement of STAT5 signalling.

Extensive phosphoprotein profiling revealed potential involvement of heat shock protein 90 (HSP90), ABL1, NFкB p50 and oestrogen receptor- $\alpha$ in the effects of FNDC4 on macrophages and further supported a role for the ERK/p38 pathway (MKK3, MSK1, MAPKAPK2, SHP-2) downstream of FNDC4. Interestingly, the $\mathrm{BCR}-\mathrm{ABL}$ complex was shown to suppress HSP90 phosphorylation at Ser226, resulting in a reduction of apoptosome function and thus improved cell survival ${ }^{38}$. Although HSP90 inhibition was shown to dampen inflammation in vitro 39 and in vivo ${ }^{40,41}$, the role of Ser226 phosphorylation of HSP90 in inflammation in macrophages is unclear. We observed an upregulation of Ser337 phosphorylation of NFKB p105/p50. $\mathrm{NF} \kappa \mathrm{B}$ p50 is constitutively phosphorylated by PKAc at Ser337 (ref. 42) and in contrast to p50/p65 heterodimers that are mostly involved in proinflammatory processes, p50 homodimers are mostly bound to DNA in resting cells where they maintain negative regulation of $\mathrm{NF} \kappa \mathrm{B}$ gene expression ${ }^{42}$. Finally, it has been well established that oestrogen receptors have an important role in modulating anti-inflammatory processes ${ }^{43}$.

In conclusion, we have characterized a novel secreted factor, FNDC4, targeting macrophages. Fndc4 is upregulated with inflammation in human IBD and in several mouse models for inflammatory diseases. FNDC4 treatment of macrophages improved cell survival, downregulated proinflammatory gene expression and strongly reduced phagocytosis. FNDC4 supplementation in vivo attenuated DSS-induced colitis, indicating that FNDC4 has therapeutic potential. The use of a human Fc-bound recombinant FNDC4 protein ensures prolonged in vivo stability of the protein and may have the propensity for direct use in humans.

\section{Methods}

Gene expression analysis. RNA-extraction was performed by trizol extraction followed by cleanup using the RNeasy system (Qiagen, Venlo, The Netherlands). Subsequently, complementary DNA synthesis was performed using the High Capacity Reverse Transcription Kit from Applied Biosystems (Life Technologies, Bleiswijk, The Netherlands) following the manufacturer's instructions. RT-qPCR was performed using the iTaq Universal SYBR Green Supermix (Bio-Rad, Solna, Sweden) on a ViiA7 Real-Time PCR System (Life Technologies) and quantified using the $\triangle \triangle \mathrm{Ct}$ method where $T B P$ was used as housekeeping gene. In the DSS-induced colitis model, RNA was purified with lithium chloride as described previously ${ }^{44}$, since DSS inhibits reverse transcriptases and polymerases ${ }^{44}$. Primer sequences are listed in Supplementary Table 1. Affymetrix global gene expression analysis (MoGene-1_1-st-v1 arrays) was performed in the BEA core facility of Karolinska Institutet. Three samples per group were used and genes displaying probe intensity $>100$ and $P$ values $<0.05$ using Student's $T$-tests were selected for downstream analysis. Furthermore, $q$ values were calculated (corrected for multiple testing). Microarray data have been deposited in NCBI's Gene Expression Omnibus and are accessible through GEO series accession number GSE76172.

Animal experiments. All animal procedures were in compliance with protocols approved by local government authorities (Institutional Animal Care and Use Committee of Karolinska Institutet, Stockholm, Sweden).

Male $\mathrm{C} 57 \mathrm{Bl} / 6 \mathrm{~N}$ mice aged 10-14 weeks were housed under specific-pathogenfree (SPF) conditions in groups of 3 to 10 in a 12-h-12-h light-dark cycle (with lights on at 06:00 hours) and fed standard chow diet.

Colitis was induced by administration of 3\% DSS (MW $40 \mathrm{kDa}$, \#DB001, TdB Consultancy, Uppsala, Sweden) with the drinking water, provided ad libitum for 5 days. The non-treated control group received tap water only. $2.7 \mathrm{mg} \mathrm{kg}^{-1}$ body weight hFc- FNDC4 or hFc control protein (amount matched for molecular weight) was injected on days 0, 2 and 4. Body weight was measured daily. The disease activity index was based on Cooper et al. ${ }^{12}$ It is a combined score of stool consistency, intestinal bleeding and weight loss. 
Glomerulonephritis was induced by the administration of antiserum raised against the glomerular basement membrane (Probetex, San Antonio, Texas, USA). $200 \mu \mathrm{l}$ of serum was injected intraperitoneally. Mice were sacrificed 5 days post injection.

Transient ischaemia reperfusion injury of the kidney was performed as described previously ${ }^{45}$

Fndc4 $\mathrm{KO}$ mice were generated by crossing mice harbouring lox $\mathrm{P}$ sites flanking exons 2-5 of the Fndc4 locus with CMV-Cre-recombinase expressing mice (B6.C-Tg(CMV-cre) $1 \mathrm{Cgn} / \mathrm{J}$, Jackson Laboratories), resulting in Fndc4 KO mice on a mixed $\mathrm{C} 57 \mathrm{Bl} / 6 \mathrm{NJ}$ background. Fndc4 $\mathrm{KO}$ mice were born with the expected Mendelian ratio, had no detectable developmental defects, and were fertile with a normal litter size.

Human IBD cohort. During routine endoscopy in 52 adult patients investigated for a known IBD diagnosis or in the work-up for suspected gastrointestinal disorders (Supplementary Table 2), colorectal and ileal mucosal biopsy specimens were collected. Nineteen patients not afflicted with IBD and without intestinal inflammation or pathological findings were included as non-inflamed non-IBD controls. Study biopsies were collected in parallel to, and from the same locations as biopsies for histopathologic assessment. Each biopsy was categorized as 'inflamed' or 'non-inflamed' based on a composite evaluation of macroscopic findings assessed by one experienced endoscopist (S.A.) and the routine histopathologic assessment.

Biopsy specimens for RNA purification were immersed in RNAlater RNA stabilization reagent (Qiagen, Hilden, DE) and stored at $4{ }^{\circ} \mathrm{C}$ overnight, and thereafter at $-20^{\circ} \mathrm{C}$ awaiting RNA purification.

This part of the study was approved by the Regional Ethical Review Board in Linköping, Sweden (Dnr 2011/201-31). Written informed consent was obtained from all participants.

Cell culture. Bone marrow-derived macrophages were generated from C57Bl/6N mice as previously described ${ }^{46}$. Macrophages were cultured in DMEM supplemented with $10 \%$ FCS, $1 \%$ PenStrep (all from Gibco/Life Technologies, Bleiswijk, The Netherlands) and 20\% conditioned media collected from L929 cells. Before experiments, cells were starved in 1\% FCS DMEM for $24 \mathrm{~h}$. Further treatments were also performed in starvation media.

For STAT3 inhibition, cells were incubated with $50 \mu \mathrm{M}$ S3I-201 (Santa Cruz Biotechnology, Dallas, Texas, USA).

Peripheral blood mononuclear cells were isolated from buffy-coated blood obtained from healthy blood donors using Ficoll-Hypaque gradient centrifugation (BD Diagnostics, Franklin Lakes, NJ), followed by monocyte isolation using the EasySep Human monocyte enrichment kit without CD16 depletion (StemCell Technologies, Vancouver, Canada), according to manufacturers protocols. $0.5-1.0 \times 10^{6}$ monocytes per well were plated in six-well plates with complete RPMI media supplemented with $50 \mathrm{ng} \mathrm{ml}^{-1}$ colony-stimulating factor-1 (Biolegend, San Diego, CA) for 8 days to generate macrophages.

Fndc4 promoter luciferase assay. McArdle cells were transfected with a pGl3 reporter vector (Promega) containing the mouse Fndc4 upstream promoter region using Lipofectamine 2000 (Life Technologies) as transfection reagent. After $8 \mathrm{~h}$, cells were treated with TGF- $\beta 1$ ( $20 \mathrm{ng} \mathrm{ml}^{-1}$; Peprotech, Stockholm, Sweden) and/or SB-431542 (20 $\mathrm{MM}$; Merck Millipore) for $20 \mathrm{~h}$. The luciferase assay (Dual-Glo, Promega) was performed according to the manufactures instructions. Values are reported relative to the non-treated control group.

Production of Fc-fusion proteins. His-Fc-FNDC4 fusion protein was expressed using pEFIRES expression vector ${ }^{47}$. The DNA fragment coding signal peptide (SP) from Fndc5 fused to 6 His-FC was synthesized by GeneScript, Inc., USA, and cloned into the pEFIRES modified multiple cloning site using NheI and NotI restriction sites. The extracellular domain of the Fndc4 gene was PCR-amplified using mouse clone MR223815 (Origene, Rockville, MD, USA) as a template and set of primers: forward: 5'-GAGAGCGGCCGCTCGACCTCCCTCTCCTGTG-3'; reverse: $5^{\prime}$-GAGAGAATTCATTCCCCTGTCTGCAATGGC-3'. The amplified Fndc4 fragment was cloned into an SP-6His-Fc-pEFIRES vector using NotI and EcoRI restriction sites to produce an SP-6His-Fc-Fndc4-pEFIRES expression construct. These constructs were transfected to CHO-S cells using Lipofectamine 2000 (Life Technologies) and stably producing cell lines were selected using puromycin (Sigma-Aldrich, St Louis, MO, USA) as selection agent. For protein production stable CHO-S suspension cultures were grown in CD OptiCHO medium (Life Technologies) supplemented with Ala-Glu (Sigma-Aldrich). Culture supernatants were loaded onto HisTrap Excel columns (GE Healthcare, Stockholm, Sweden) in $5 \mathrm{mM}$ imidazole containing buffer, washed with $20 \mathrm{mM}$ imidazole and eluted with $400 \mathrm{mM}$ imidazole. Eluted protein was dialyzed against $3 \times$ PBS buffer. Purity of the produced recombinant protein was about 95\% (based on Coomassie staining). The protein concentration was measured using the BCA Protein Assay Kit (Pierce Biotechnology, Inc./Thermo Fischer Scientific, Rockford, IL, USA). Protein purity was assessed by SDS-PAGE and Coomassie staining using PageBlue Protein Staining Solution (Thermo Fisher Scientific). Kinetics of the recombinant protein were assessed by analysis of plasma protein content and stability.
hFc-FNDC4 or hFc control protein was injected into 9- to 10 -week-old C57Bl/6 mice (single injection of $3 \mathrm{mg} \mathrm{kg}^{-1}$ body weight). Plasma was collected after $24 \mathrm{~h}$ and 5 days and boiled in reducing buffer for $7 \mathrm{~min}$. $0.2 \mu \mathrm{l}$ of plasma per sample was blotted against hFc (IgG). Recombinant hFc and hFc-FNDC4 was spiked into plasma and treated in the same way as the experimental samples to serve as positive controls.

Analysis of FNDC4 secretion from HEK293 cells. The FNDC4 N-terminal FLAG, C-terminal Myc construct was created by modifying a C-terminally tagged FNDC4 mouse complementary DNA clone purchased from Origene (MC210221). The full-length, C-terminally modified FNDC4 was first cloned into the pENTR-1a dual-selection vector (Invitrogen). The N-terminal FLAG tag was subsequently inserted between FNDC4 S49 and P50 via site-directed mutagenesis. The C-terminal FLAG tag was removed by introducing a stop codon directly after the C-terminal Myc, also by site-directed mutagenesis, resulting in an FNDC4 construct with an N-terminal FLAG and a C-terminal Myc. The site-directed mutagenesis was performed using the QuikChange XL Site-Directed Mutagenisis Kit (Agilent). The resulting construct was then recombined with the pT-RExDEST30 gateway vector from Invitrogen for transient expression in mammalian cell lines.

HEK293 cells were cultured in DMEM-GlutaMAX supplemented with 10\% fetal bovine serum and 1\% PenStrep (Invitrogen). Transient transfections were performed using Lipofectamine 2000 (Invitrogen) in accordance with the manufacturer's instructions. Transfected cells were cultured for $24 \mathrm{~h}$, washed and subsequently switched to serum-free FreeStyle 293 Expression Media (Gibco). The conditioned FreeStyle media and cell lysates were harvested after $24 \mathrm{~h}$, and media was filtered and concentrated $20 \times$, after which both cell lysates and media were probed using western blot.

FNDC4 binding to immune cells. Blood was collected from FcgR-deficient mice (Fcg/2b DKO) on C57BL/6 background and red blood cells were lysed with two rounds of RBC lysis buffer (BioLegend, San Diego, CA, USA). Peritoneal macrophages were collected from the same mice by lavage from the peritoneal cavity using $5 \mathrm{ml}$ ice cold PBS. Cells were dissolved in FACS buffer (PBS $+3 \% \mathrm{FBS}$ ) containing hFc-FNDC4 or hFc control $(0.5 \mu \mathrm{M})$ and incubated $1 \mathrm{~h}$ in room temperature, washed, followed by $1 \mathrm{~h}$ incubation with an anti-human IgG secondary antibody conjugated to PE (Life Technologies, H10104, 1:200) and washing steps. Subsequently, cells were labelled with antibodies to define cell populations (CD11b, B220, TCRb, GR-1, CD123, NK1.1 and F4/80). After washings, cells were collected on a BD LSRII FACS and analysed by FlowJo software. Dead cells were excluded on the basis of labelling with 7-AAD and duplicates excluded by a FSC-H FSC-A gate. Quantification was performed by defining the mean fluorescence intensity of PE on gated cell populations.

RNA in situ hybridization. In situ RNA hybridization for Fndc4 mRNA was performed on formaldehyde-fixed, paraffin-embedded colonic tissue using RNAscope technology (Advanced Cell Diagnostics, Hayward, CA, USA) with custom-made probes for mouse Fndc4, following the manufacturer's instructions (RNAscope 2.0 HD red detection kit). Probes targeting $U b c$ and Dabp were used as positive and negative control probes, respectively. Slides were counterstained with hematoxylin.

Histopathology. Renal tissue was stained with periodic acid Schiff s stain for histological analysis. To examine the glomerular injury, the glomerular damage index (0: none, 1: slight, 2: moderate, 3 : severe) was determined by assessing glomerular cellularity, mesangiolysis, glomerular expansion and crescent formation. Crescent formation was defined as the presence of three or more layers of cells in Bowman's space.

Colon pathology was assessed as described previously ${ }^{48}$. Briefly, severity of lesion was graded as follows: $0=$ normal, $1=$ mild, $2=$ moderate, $3=$ severe. Mild lesions were small, focal or widely separated multifocal areas of inflammation limited to the lamina propria. Moderate lesions were either multifocal or represented by locally extensive areas of inflammation extending into the submucosa. Severe lesions were ulcers covering large areas $(>20$ crypts) of the mucosa.

Western blotting. Western blots were performed with cell lysates in RIPA-lysis buffer mixed 1:1 with Laemmli sample buffer (Bio-Rad, Mississauga, Ontario, Canada) and processed for standard SDS-PAGE and western blotting. Protein concentration was determined and equal amounts of protein were loaded per lane. The nitrocellulose membranes were incubated with antibodies directed against phosphoERK (1:2,000; Thr202, Tyr204; \#9101; Cell Signaling, Leiden, The Netherlands), ERK (1:2,000; \#4695; Cell Signaling), phosphoSTAT3 (Tyr705) (1:1,000; \#9145; Cell Signaling), STAT3 (1:500; \#sc-482; Santa Cruz Biotechnology, Dallas, Texas, USA) and phosphoSTAT6 (1:1,000; \#ab54461; Abcam, Cambridge, UK). Detection of phosphorylation versus total protein levels (pERK/ERK, pSTAT3/STAT3) was performed on separate gels (no stripping). Antibody binding was revealed by incubation with donkey anti-rabbit horseradish peroxidase-linked 
$\operatorname{IgG}(1: 10,000)$ (\#97085; Abcam) and the SuperSignal West Femto immunoblotting detection system (Thermo Scientific). Chemiluminescence was detected by autoradiography using Amersham Hyperfilm ECL (GE Healthcare, Uppsala, Sweden). Images have been cropped for presentation. Full-size images are presented in Supplementary Fig. 11.

Phagocytosis assay. Alexa Fluor 488 conjugated zymosan A particles (Life Technologies, Stockholm, Sweden) were diluted in starvation media to obtain a concentration of $2 \mathrm{E} 6$ particles per $\mathrm{ml}$. Bone marrow macrophages were incubated with the zymosan particles for 30 min followed by five washes in PBS to wash away non-phagocytosed particles. On fixation in $4 \%$ formaldehyde, nuclei were stained with DAPI and cells were imaged using a Leica TCS SP8 confocal laser-scanning microscope (Leica Microsystems, Kista, Sweden).

Apoptotic cell clearance assay. Dead cell clearance was performed as described earlier ${ }^{49}$. Briefly, bone marrow macrophages were treated with $\mathrm{hFc}$-FNDC4 or hFc control for $24 \mathrm{~h}$ before incubation with CellTracker Orange CMTMR (Life Technologies)-labelled apoptotic thymocytes (dexamethasoneinduced). Phagocytosis of apoptotic thymocytes was assessed after $1 \mathrm{~h}$.

NO assay. NO production was assessed in cell culture media using the nitrate/ nitrite calorimetric assay from Cayman Chemicals (Ann Arbour, Michigan, USA).

Flow cytometry. After separation from the small intestine the colon was cut longitudinally and one half was collected in ice-cold PBS for flow cytometry analysis. The colon was further cut into $1 \mathrm{~mm}$ pieces. Subsequently, colon pieces were incubated in magnesium and calcium-free HBSS (Life Technologies) containing $1 \mathrm{mM}$ dithiothreitol and $5 \mathrm{mM} \mathrm{EDTA}$ at $37^{\circ} \mathrm{C}$ for $20 \mathrm{~min}$ with shaking, after which the EDTA- and DTT-containing buffer was refreshed followed by another $20 \mathrm{~min}$. incubation at $37^{\circ} \mathrm{C}$. Then, colon pieces were treated with $20 \mu \mathrm{ml}^{-1}$ collagenase D and $2 \%$ DNAse I (Sigma-Aldrich) for $45 \mathrm{~min}$ at $37^{\circ} \mathrm{C}$ with shaking. Single cells were obtained by filtering through a $100 \mu \mathrm{m}$ cell strainer. Samples were centrifuged for $5 \mathrm{~min}$. at $600 \mathrm{~g}$ to pellet cells. Subsequently, cell pellets were resuspended in FACS buffer (PBS supplemented with 3\% FCS and $10 \mathrm{mM}$ EDTA) containing Fc block, 1:200 (\#553141; BD Biosciences). Cells were incubated for $15 \mathrm{~min}$. at room temperature, followed by a wash in FACS buffer. Subsequently, cells were incubated with an antibody cocktail containing CD45.2-FITC (\#109806, BioLegend) and CD11b-PE (\#557397, BD Biosciences) at 1:200 for $30 \mathrm{~min}$ on ice. Cells were washed once and resuspended in FACS buffer containing 1:30 diluted 7-AAD staining solution (BioLegend) to determine cell viability. Samples were filtered through $50 \mu \mathrm{m}$ nylon gauze before analysis on a FACSCanto II with FACSDiva software (BD Biosciences). Unstained and single-stained control samples were included in the experiments. Acquired data were gated and analysed with FlowJo software (TreeStar, Inc., Ashland, OR, USA).

Phosphoprotein profiling. Cell lysates were obtained from primary bone marrow macrophages treated for $30 \mathrm{~min}$ with $\mathrm{hFc}-\mathrm{FNDC} 4$ or $\mathrm{hFc}$ control protein $(\mathrm{N}=2$ per condition). The Phospho Explorer antibody microarray, which was designed and manufactured by Full Moon Biosystems, Inc. (Sunnyvale, CA), contains 1,318 antibodies. Each of the antibodies has two replicates that are printed on a coated glass microscope slide, along with multiple positive and negative controls. The antibody array experiment was performed by Full Moon Biosystems, according to their established protocols ${ }^{50}$

The slides were scanned on an Axon GenePix array scanner, and the images were analysed with GenePix Pro 6.0 (Molecular Devices, Sunnyvale, CA). The fluorescence signal $(I)$ of each antibody was obtained from the fluorescence intensity of this antibody spot after subtraction of the blank signal (spot in the absence of antibody). For each phospho-specific antibody, a phosphorylation signal ratio was calculated.

ChIP assay for analysis of STAT3 DNA binding. Bone marrow-derived macrophages were starved overnight followed by $6 \mathrm{~h}$ treatment with $200 \mathrm{nM} \mathrm{hFc}$-FNDC4 or hFc control. Cells were crosslinked for 10 min with $1 \%$ formaldehyde, followed by quenching for $5 \mathrm{~min}$ with $12.5 \mathrm{mM}$ glycine. Cells were further processed for ChIP analysis using the Magna ChIP A/G chromatin immunoprecipitation kit (Millipore,Solna, Sweden), following the manufacturer's instructions. Briefly, after cell and nucleus lysis, chromatin was sheared 10 times (20 s on and $30 \mathrm{~s}$ off) with a Bioruptor sonicator (Diagenode, Seraing, Belgium) to yield fragment sizes of $100-500$ base pairs. After $10 \%$ of the sample was saved as 'input control', sheared chromatin was immunoprecipitated overnight with antibody to STAT3 (\#sc-482; Santa Cruz Biotechnology) and normal rabbit IgG (Sigma-Aldrich) as negative control, followed by $1 \mathrm{~h}$ of incubation at $4{ }^{\circ} \mathrm{C}$ with Protein $\mathrm{A} / \mathrm{G}$ magnetic beads. After washing and reverse crosslinking, DNA was purified and analysed using iTaq Universal SYBR green supermix (Life Technologies, Stockholm, Sweden) on a ViiA7 Real-Time PCR System (Life Technologies). Primers sequences for RT-qPCR analysis of binding to Socs 3 were: $5^{\prime}$-CCCCCAACTTCTCATTCACA- $3^{\prime}$ (forward) and $5^{\prime}$-TACATGAGGACCTCGGAGTG-3' (reverse) ${ }^{51}$. Promoter occupancy was calculated as percentage of input DNA.

Statistics. Differences between groups were evaluated using two-tailed unpaired or paired (IBD cohort) Student's T-tests for single comparisons or one-way analysis of variances in combination with Tukey's post hoc tests for multiple comparisons when data was normally distributed, and Mann-Whitney rank tests for nonnormally distributed data (scores). The Benjamini-Hochberg procedure was used to correct for multiple testing for experiments where gene expression of a large panel of genes was shown. In these cases, the level of significance was set at false discovery rate $(q)=0.10$. Correlations were evaluated using Pearson's correlation coefficients. IBM SPSS Statistics version 22 and GraphPad Prism version 6 were used for statistical analyses. $P<0.05$ was considered statistically significant.

\section{References}

1. Molodecky, N. A. et al. Increasing incidence and prevalence of the inflammatory bowel diseases with time, based on systematic review. Gastroenterology 142, 46-54 e42; quiz e30 (2012).

2. Podolsky, D. K. Inflammatory bowel disease. N. Engl. J. Med. 347, 417-429 (2002).

3. Steinbach, E. C. \& Plevy, S. E. The role of macrophages and dendritic cells in the initiation of inflammation in IBD. Inflamm. Bowel Dis. 20, 166-175 (2014).

4. Renna, S., Cottone, M. \& Orlando, A. Optimization of the treatment with immunosuppressants and biologics in inflammatory bowel disease. World J. Gastroenterol. 20, 9675-9690 (2014).

5. Teufel, A., Malik, N., Mukhopadhyay, M. \& Westphal, H. Frcp1 and Frcp2, two novel fibronectin type III repeat containing genes. Gene 297, 79-83 (2002).

6. Boström, P. et al. A PGC1- $\alpha$-dependent myokine that drives brown-fat-like development of white fat and thermogenesis. Nature 481, 463-468 (2012).

7. Wrann, C. D. et al. Exercise induces hippocampal BDNF through a PGC-1alpha/FNDC5 pathway. Cell Metab. 18, 649-659 (2013).

8. Jedrychowski, M. P. et al. Detection and quantitation of circulating human irisin by tandem mass spectrometry. Cell Metab. 22, 734-740 (2015).

9. Wang, F. et al. RNAscope: a novel in situ RNA analysis platform for formalin-fixed, paraffin-embedded tissues. J. Mol. Diagn. 14, $22-29$ (2012).

10. Heng, T. S. \& Painter, M. W. The Immunological Genome Project: networks of gene expression in immune cells. Nat. Immunol. 9, 1091-1094 (2008).

11. Tiano, J. P., Springer, D. A. \& Rane, S. G. SMAD3 negatively regulates serum irisin and skeletal muscle FNDC5 and PGC-1alpha during exercise. J. Biol. Chem. 290, 7671-7684 (2015).

12. Cooper, H. S., Murthy, S. N., Shah, R. S. \& Sedergran, D. J. Clinicopathologic study of dextran sulfate sodium experimental murine colitis. Lab. Invest. 69, 238-249 (1993).

13. Stevceva, L., Pavli, P., Husband, A. J. \& Doe, W. F. The inflammatory infiltrate in the acute stage of the dextran sulphate sodium induced colitis: B cell response differs depending on the percentage of DSS used to induce it. BMC Clin. Pathol. 1, 3 (2001)

14. Takai, T., Li, M., Sylvestre, D., Clynes, R. \& Ravetch, J. V. FcR gamma chain deletion results in pleiotrophic effector cell defects. Cell 76, 519-529 (1994).

15. Takai, T., Ono, M., Hikida, M., Ohmori, H. \& Ravetch, J. V. Augmented humoral and anaphylactic responses in Fc gamma RII-deficient mice. Nature 379, 346-349 (1996).

16. Zhang, Y. et al. Irisin stimulates browning of white adipocytes through mitogen-activated protein kinase $\mathrm{p} 38$ MAP kinase and ERK MAP kinase signaling. Diabetes 63, 514-525 (2014).

17. Zigmond, E. et al. Ly6C hi monocytes in the inflamed colon give rise to proinflammatory effector cells and migratory antigen-presenting cells. Immunity 37, 1076-1090 (2012).

18. Biswas, S. K., Chittezhath, M., Shalova, I. N. \& Lim, J. Y. Macrophage polarization and plasticity in health and disease. Immunol. Res. 53, 11-24 (2012).

19. Jacoby, J. J. et al. Cardiomyocyte-restricted knockout of STAT3 results in higher sensitivity to inflammation, cardiac fibrosis, and heart failure with advanced age. Proc. Natl Acad. Sci. USA 100, 12929-12934 (2003).

20. Grant, L. et al. Myeloid-cell protein tyrosine phosphatase-1B deficiency in mice protects against high-fat diet and lipopolysaccharide induced inflammation, hyperinsulinemia and endotoxemia through an IL10 STAT3 dependent mechanism. Diabetes 63, 456-470 (2013).

21. Moon, H.-S., Dincer, F. \& Mantzoros, C. S. Pharmacological concentrations of irisin increase cell proliferation without influencing markers of neurite outgrowth and synaptogenesis in mouse H19-7 hippocampal cell lines. Metabolism 62, 1131-1136 (2013).

22. Arranz, A. et al. Akt1 and Akt2 protein kinases differentially contribute to macrophage polarization. Proc. Natl Acad. Sci. USA 109, 9517-9522 (2012)

23. Ghia, J. E., Blennerhassett, P., Kumar-Ondiveeran, H., Verdu, E. F. \& Collins, S $M$. The vagus nerve: a tonic inhibitory influence associated with inflammatory bowel disease in a murine model. Gastroenterology 131, 1122-1130 (2006). 
24. Smith, P. et al. Infection with a helminth parasite prevents experimental colitis via a macrophage-mediated mechanism. J. Immunol. 178, 4557-4566 (2007).

25. Tokuyama, H. et al. The simultaneous blockade of chemokine receptors CCR2, CCR5 and CXCR3 by a non-peptide chemokine receptor antagonist protects mice from dextran sodium sulfate-mediated colitis. Int. Immunol. 17, 1023-1034 (2005)

26. Chami, B., Yeung, A. W., van Vreden, C., King, N. J. \& Bao, S. The role of CXCR3 in DSS-induced colitis. PLoS ONE 9, e101622 (2014).

27. Uza, N. et al. SR-PSOX/CXCL16 plays a critical role in the progression of colonic inflammation. Gut 60, 1494-1505 (2011).

28. Murray Peter, J. et al. Macrophage activation and polarization: nomenclature and experimental guidelines. Immunity 41, 14-20 (2014).

29. Xue, J. et al. Transcriptome-based network analysis reveals a spectrum model of human macrophage activation. Immunity 40, 274-288 (2014).

30. Zhou, J. et al. CXCR3-dependent accumulation and activation of perivascular macrophages is necessary for homeostatic arterial remodeling to hemodynamic stresses. J. Exp. Med. 207, 1951-1966 (2010).

31. Geletu, M., Guy, S., Arulanandam, R., Feracci, H. \& Raptis, L. Engaged for survival: from cadherin ligation to STAT3 activation. JAKSTAT 2, e27363 (2013).

32. Murray, P. J. Understanding and exploiting the endogenous interleukin-10/ STAT3-mediated anti-inflammatory response. Curr. Opin. Pharmacol. 6, 379-386 (2006)

33. Takeda, K. et al. Enhanced Th1 activity and development of chronic enterocolitis in mice devoid of Stat 3 in macrophages and neutrophils. Immunity 10, 39-49 (1999).

34. Atreya, R. et al. Blockade of interleukin 6 trans signaling suppresses T-cell resistance against apoptosis in chronic intestinal inflammation: evidence in crohn disease and experimental colitis in vivo. Nat. Med. 6, 583-588 (2000).

35. Chaudhry, A. et al. CD $4+$ regulatory T cells control TH17 responses in a Stat3-dependent manner. Science 326, 986-991 (2009).

36. Willson, T. A. et al. STAT3 genotypic variation and cellular STAT3 activation and colon leukocyte recruitment in pediatric Crohn disease. J. Pediatr. Gastroenterol. Nutr. 55, 32-43 (2012).

37. Polgar, N. et al. Investigation of JAK2, STAT3 and CCR6 polymorphisms and their gene-gene interactions in inflammatory bowel disease. Int. J. Immunogenet. 39, 247-252 (2012).

38. Kurokawa, M., Zhao, C., Reya, T. \& Kornbluth, S. Inhibition of apoptosome formation by suppression of Hsp90beta phosphorylation in tyrosine kinase-induced leukemias. Mol. Cell. Biol. 28, 5494-5506 (2008).

39. Shimp, III S. et al. HSP90 inhibition by 17-DMAG reduces inflammation in J774 macrophages through suppression of Akt and nuclear factor- $\kappa \mathrm{B}$ pathways. Inflamm. Res. 61, 521-533 (2012).

40. Chatterjee, A. et al. Heat shock protein 90 inhibitors prolong survival, attenuate inflammation, and reduce lung injury in murine sepsis. Am. J. Respir. Crit. Care Med. 176, 667-675 (2007)

41. Madrigal-Matute, J. et al. Heat shock protein 90 inhibitors attenuate inflammatory responses in atherosclerosis. Cardiovasc. Res. 86, 330-337 (2010).

42. Guan, H., Hou, S. \& Ricciardi, R. P. DNA Binding of repressor Nuclear Factor $-\kappa \mathrm{B}$ p50/p50 depends on phosphorylation of Ser337 by the protein kinase A catalytic subunit. J. Biol. Chem. 280, 9957-9962 (2005).

43. Härkönen, P. L. \& Väänänen, H. K. Monocyte-macrophage system as a target for estrogen and selective estrogen receptor modulators. Ann. NY Acad. Sci. 1089, 218-227 (2006)

44. Viennois, E., Chen, F., Laroui, H., Baker, M. T. \& Merlin, D. Dextran sodium sulfate inhibits the activities of both polymerase and reverse transcriptase: lithium chloride purification, a rapid and efficient technique to purify RNA. BMC Res. Notes 6, 360 (2013).

45. Iwata, Y. et al. Aberrant macrophages mediate defective kidney repair that triggers nephritis in lupus-susceptible mice. J. Immunol. 188, 4568-4580 (2012).
46. Menke, J. et al. Circulating CSF-1 promotes monocyte and macrophage phenotypes that enhance lupus nephritis. J. Am. Soc. Nephrol. 20, 2581-2592 (2009).

47. Hobbs, S. M. Expression of recombinant antibody fusion proteins in E. coli. Methods Mol. Biol. 80, 419-425 (1998).

48. Bleich, A. et al. Refined histopathologic scoring system improves power to detect colitis QTL in mice. Mamm. Genome 15, 865-871 (2004).

49. Khanna, S. et al. Macrophage dysfunction impairs resolution of inflammation in the wounds of diabetic mice. PLoS ONE 5, e9539 (2010).

50. Bernier, M. et al. Negative regulation of STAT3 protein-mediated cellular respiration by SIRT1 protein. J. Biol. Chem. 286, 19270-19279 (2011).

51. Hutchins, A. P. et al. Distinct transcriptional regulatory modules underlie STAT3's cell type-independent and cell type-specific functions. Nucleic Acids Res. 41, 2155-2170 (2013).

\section{Acknowledgements}

We thank Dr Xiaochuan Ma for assistance in testing the hFc-FNDC4 protein batches, Lena Svensson for handling of samples from IBD patients and controls, Dr Stephanie Zwicker for providing human macrophages, and Dr Raoul Kuiper and Dr Björn Rozell for histopathology assessments. We thank Professor Thomas Perlmann, Professor Urban Lendahl and Professor Camilla Sjögren for critical reading of the manuscript. This research was partly funded by an ERC Starting Grant (309596) as well as by grants from the Wenner Gren Foundation, Vetenskapsrådet (M23/11), Ragnar Söderbergs Stiftelse, Svenska Sällskapet för Medicinsk Forskning and the Hållsten Academy (to P.A.B.) and a grant from Karolinska Institutet's Research Foundation (2014fobi41721) (to M.B.). This work was further supported by funds from Vetenskapsrådet (the Swedish Research Council) (523-2012-2480) and Stockholm County Council/ALF project (20140367) (to E.A.B.); the German Research Foundation (DFG, Ge2386/1-1) and the Swedish Cancer Foundation (Cancerfonden, 2014/1376) (to M.G.); FORSS, the Medical Research Council of South-Eastern Sweden (236541-2012), Bengt Ihre-Fonden (2012-SLS 254491), and Karolinska Institutet's Research Foundation (2014fobi42063) (to S.A.), and by a grant from the Center of Innovative Medicine, CIMED (to R.T.).

\section{Author contributions}

M.B. designed and performed experiments, analysed the data and wrote the manuscript. M.G., F.W. and E.A.B. contributed to experiments and revised/edited the manuscript. J.P., A.G., O.S., E.G. and Y.I. contributed to experiments. S.A. recruited the IBD patients and controls, performed all endoscopies and revised the manuscript. J.S. was involved in the organization and analysis of the IBD cohort. R.T. was involved in the discussion and revised/edited the manuscript. P.A.B. designed experiments, analysed the data and revised/edited the manuscript.

\section{Additional information}

Supplementary Information accompanies this paper at http://www.nature.com/ naturecommunications

Competing financial interests: The authors declare no competing financial interests

Reprints and permission information is available online at http://npg.nature.com/ reprintsandpermissions/

How to cite this article: Bosma, M. et al. FNDC4 acts as an anti-inflammatory factor on macrophages and improves colitis in mice. Nat. Commun. 7:11314 doi: $10.1038 /$ ncomms11314 (2016).

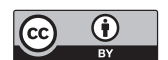

This work is licensed under a Creative Commons Attribution 4.0 International License. The images or other third party material in this article are included in the article's Creative Commons license, unless indicated otherwise in the credit line; if the material is not included under the Creative Commons license, users will need to obtain permission from the license holder to reproduce the material. To view a copy of this license, visit http://creativecommons.org/licenses/by/4.0/ 